\title{
FDIA System for Sensors of the Aero-Engine Control System Based on the Immune Fusion Kalman Filter
}

\author{
Linfeng Gou, Ruiqian Sun (D), and Xiaobao Han \\ Northwestern Poly-Technical University, School of Power and Energy, Xi'an 710129, China \\ Correspondence should be addressed to Ruiqian Sun; sunruiqian@mail.nwpu.edu.cn
}

Received 6 November 2020; Revised 25 January 2021; Accepted 2 March 2021; Published 19 March 2021

Academic Editor: Chiara Boccaletti

Copyright ( 92021 Linfeng Gou et al. This is an open access article distributed under the Creative Commons Attribution License, which permits unrestricted use, distribution, and reproduction in any medium, provided the original work is properly cited.

\begin{abstract}
The Kalman filter plays an important role in the field of aero-engine control system fault diagnosis. However, the design of the Kalman filter bank is complex, the structure is fixed, and the parameter estimation accuracy in the non-Gaussian environment is low. In this study, a new filtering method, immune fusion Kalman filter, was proposed based on the artificial immune system (AIS) theory and the Kalman filter algorithm. The proposed method was used to establish the fault diagnosis, isolation, and accommodation (FDIA) system for sensors of the aero-engine control system. Through a filtering calculation, the FDIA system reconstructs the measured parameters of the faulty sensor to ensure the reliable operation of the aero engine. The AIS antibody library based on single sensor fault was constructed, and with feature combination and library update, the FDIA system can reconstruct the measured values of multiple sensors. The evaluation of the FDIA system performance is based on the Monte Carlo method. Both steady and transient simulation experiments show that, under the non-Gaussian environment, the diagnosis and isolation accuracy of the immune fusion Kalman filter is above 95\%, much higher than that of the Kalman filter bank, and compared with the Kalman particle filter, the reconstruction value is smoother, more accurate, and less affected by noise.
\end{abstract}

\section{Introduction}

Aero engine is a highly complex thermodynamic system, working in the harsh environment of high temperature, high pressure, and high rotating speed. As the brain of the aero engine, the control system plays an irreplaceable role in the normal operation of the aero engine. The aero-engine control system is mainly composed of electronic controller, sensors, actuators, etc. According to the statistical analysis of big data, more than $90 \%$ of engine faults are sensor faults. Therefore, how to timely diagnosis the fault, accurately isolate the fault sensor, and propose accommodation has always been a research hotspot. The FDIA system can guarantee the safe operation of aero engines and reduce the cost of maintenance, so it is indispensable for modern aero engines.

There are several methods currently available for sensor fault diagnosis in aero-engine control systems. Joly et al. used an artificial neural network to diagnose high bypass ratio military turbofan engines [1]. Kobayashi and Simon proposed a neural network hybrid genetic algorithm for engine performance evaluation [2]. Naderi and Khorasani proposed a data-driven fault detection isolation (FDI) estimation method for monitoring the aircraft gas-turbine engine sensors [3]. Xiao et al. applied a novel sliding mode fault-tolerant control method for fault diagnosis and tolerant control [4]. Yazar et al. design a FDI system based on a fullorder unknown input observer for fault detection [5]. Ogaji et al. discussed a fuzzy logic algorithm for gas-turbine fault diagnosis [6]. Lu et al. combined the particle filter with the extended Kalman filter and applied it to turbofan engine health management [7]. Kordestani applied the extended Kalman filter to the remaining life estimation and fault prognosis of aircraft components [8-10].

The above methods have certain shortcomings. For example, the design of the large closed-loop Kalman filter bank in the control system is overly complex, resulting in low computational efficiency and insufficient precision. The expert system does not readily provide workable knowledge, and the available knowledge has relatively poor integrity and 
insufficient adaptability [11]. Neural networks have structure selection, online training, and adjustment problems [12].

From the bionics' perspective, the AIS has stronger noise tolerance, fast response, and continuous learning capability. Artificial immune systems have been widely used in the field of fault diagnosis in recent years due to their powerful information-processing performance. Laurentys et al., for example, proposed a dynamic system fault diagnosis method based on the AIS [13]. Ghosh and Srinivasan used an AIS for troubleshooting and process monitoring [14]. Through theoretical analysis, Mohapatra et al. made it possible to solve the fault diagnosis in wireless sensor networks through the AIS [15]. Jiang and Chang developed a novel antibody population optimization-based AIS for rotating equipment anomaly detection [16]. In the aerospace field, Moncayo and Perhinschi were the first to apply the AIS to aircraft health management and gave detailed design plans for aircraft flight envelope reconstruction under abnormal conditions [17-22]. By taking the aircraft as the research object, they designed a distributed health management system to detect, identify, evaluate, and accommodate under abnormal conditions. The aero engine appears as a subsystem of the aircraft in the system [23]. As Moncayo and Perhinschi took airplane as the research object, only certain types of aero engine faults can be detected and located. However, as an extremely complex thermodynamic system, it is far from enough to regard the aero engine as a subsystem of the aircraft.

Aiming at the above shortcomings, this paper takes the aero-engine control system sensor as the research object and proposes a fault diagnosis method based on the immune fusion Kalman filter. Simulation experiments prove that the FDIA system based on the immune fusion Kalman filter has a good performance in sensor fault diagnosis, isolation, and accommodation. The proposed method can be used to diagnose, isolate, and reconstruct the faults not encountered in the training samples through online learning.

This paper is organized as follows. The nonlinear engine model is established and linearized in Section 2. The sensor fault model is also constructed, and a fault feature extraction method is proposed. Section 3 describes the process of establishing the FDIA system based on the immune fusion Kalman filter. Section 4 presents simulation results based on the linear model, both steady state and transient are simulated. The Monte Carlo method is used to evaluate the performance of different FDIA systems. At the same time, the accuracy of reconstruction under different noise environments is compared according to different methods. Section 5 provides concluding remarks and suggestions for further research.

\section{Aero Engine Model and Fault Feature Extraction}

The component-level model of the turbofan engine was established as presented below. The state-space model of turbofan engine was obtained by linearization. Meanwhile, the sensor fault model of the control system was established. The corresponding methods for fault feature extraction of single fault and multifault states are followed.

2.1. Aero-Engine Nonlinear Model and Linearization. A nonlinear steady-state model of a biaxial-separated exhaust turbofan engine was established in this study based on the component method. The biaxial exhaust turbofan engine is composed of intake parts, combustion parts, turbine parts, exhaust parts, and rotor parts. The intake parts include fan, low-pressure compressor (LPC), and high-pressure compressor (HPC). The combustion part consists of only the combustor. The low-pressure turbine (LPT) and highpressure turbine (HPT) constitute the turbine parts. As a separated exhaust engine, there are two exhaust channels. Correspondingly, the exhaust parts are divided into inner nozzle and outer nozzle. The rotor parts are made up of the high-pressure rotor (HPR) and low-pressure rotor (LPR). The air enters the fan after being decelerated and pressurized by the inlet. The air flow can be divided into two parts after being compressed by the fan: a part moves through the bypass, directly through the outer nozzle expanding its power as the other part moves through the LPC and HPC. A small portion of the air is used to cool the HPT and LPT, while most of the airflow enters the combustor. After combustion, the high-temperature and high-pressure gas expands through the turbine to perform the necessary work. The generated power drives the fan, LPC, and HPC and then is ultimately discharged through the inner nozzle to generate thrust. The area of the inner nozzle can be adjusted. The structure of the biaxial-separated exhaust turbofan engine is shown in Figure 1.

The engine discussed in this paper is composed of a separate model for each component. Each basic component requires multiple input variables and multiple output variables. The model includes a thermodynamic calculation formula, compressor and turbine characteristic diagram, and acceleration and deceleration characteristics of aero engine. Mathematical expressions reveal the thermodynamic relationships among various engine variables. The following assumptions were made to simplify the model:

(i) On the premise of constant flight conditions, the influence of interference input is ignored

(ii) Only the rotor of turbofan engine is considered as the energy storage component, and the volume effect is not considered

(iii) The total pressure recovery coefficient and component efficiency are considered to be unchanged within a small range

(iv) Combustion delay is ignored

The thermodynamic parameters (e.g., total temperature, total pressure, flow rate, and efficiency) in each section of the engine can be calculated according to previously published methods [24-26]. At steady-state operating points, turbofan engines meet flow balance and power balance requirements as expressed in equations (1) and (2), respectively: 


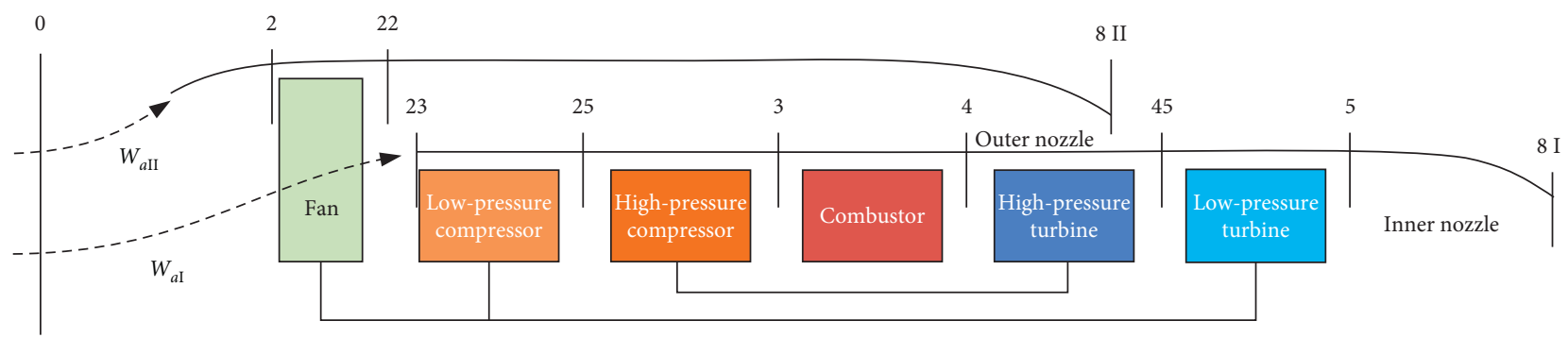

FIGURE 1: Biaxial-separated exhaust turbofan engine.

$$
\begin{aligned}
& \left\{\begin{array}{l}
W_{22}-W_{a \mathrm{I}}-W_{a \mathrm{II}}=0, \\
W_{25}-W_{23}=0, \\
W_{45}-W_{\mathrm{HTcool}}-W_{4}=0 \\
W_{5}-W_{\text {LTcool }}-W_{45}=0, \\
W_{8 \mathrm{I}}-W_{5}=0,
\end{array}\right. \\
& \left\{\begin{array}{l}
N_{\mathrm{HT}} \eta_{\text {Hspool }}-N_{\mathrm{HC}}-N_{T}=0, \\
N_{\mathrm{LT}} \eta_{\text {Lspool }}-N_{\mathrm{LC}}-N_{F}-N_{B}=0,
\end{array}\right.
\end{aligned}
$$

where $W_{22}$ is the fan outlet flow, $W_{a \mathrm{I}}$ is the flow of air through the core engine, $W_{a \text { II }}$ is the airflow through the bypass, $W_{23}$ is the LPC inlet flow, $W_{25}$ is the HPC inlet flow, $W_{4}$ is the combustor outlet flow, $W_{45}$ is the outlet flow of the HPT, $W_{5}$ is the outlet flow of the LPT, $W_{\text {HTcool }}$ is the cooling flow rate of the HPT, $W_{\text {LTcool }}$ is the cooling flow rate of the LPT, $W_{8 \mathrm{I}}$ is the core engine nozzle outlet flow, $N_{\mathrm{HT}}$ and $N_{\mathrm{LT}}$ are the output power of the HPT and LPT, respectively, $\eta_{\text {Hspool }}$ and $\eta_{\text {Lspool }}$ are the HPR and LPR efficiency, respectively, and $N_{\mathrm{HC}}, N_{\mathrm{LC}}, N_{F}, N_{T}, N_{B}$ represent the power consumed by the HPC, LPC, fans, accessories, and booster, respectively.

For the engine model established by the component method, there are seven flow and power balance equations. Seven estimation variables are thus needed to calculate the steady-state point of the nonlinear engine model. Here, the LPR rotational speed $n_{L}, \mathrm{HPR}$ rotational speed $n_{H}$, fan pressure ratio $\pi_{F}$, LPC pressure ratio $\pi_{\mathrm{LC}}$, HPC pressure ratio $\pi_{\mathrm{HC}}$, HPT pressure ratio $\pi_{\mathrm{HT}}$, and LPT pressure ratio $\pi_{\text {LT }}$ serve as estimation variables. After these seven physical quantities are introduced, the flow balance and power balance of the nonlinear engine model can be reduced to nonlinear equations in the following form:

$$
f_{j}\left(n_{L}, n_{H}, \pi_{F}, \pi_{\mathrm{LC}}, \pi_{\mathrm{HC}}, \pi_{\mathrm{HT}}, \pi_{\mathrm{LT}}\right)=0, \quad j=1,2, \ldots, 7 .
$$

The biaxial-separated exhaust turbofan engine is a multiinput and multioutput system, which falls under linear system theory. A Linear State Variable Model (LSVM) of the engine was established here accordingly. The selected $n_{L}$ and $n_{H}$ are regarded as the state variables of the system. Engine oil $w_{f}$ and nozzle throat area $A_{8}$ were selected as control variables of the system. The output variable consists of seven parameters, namely, $n_{L}, n_{H}$, LPC outlet total pressure $P_{25}$, HPC outlet total pressure $P_{3}$, HPC outlet temperature $T_{3}$,
HPT outlet total temperature $T_{45}$, and LPT export total temperature $T_{5}$.

For a complex nonlinear system, the dynamic response of the aero engine can be considered to be linear when the input varies in a small range at a given state point. Therefore, when the engine is in a certain working state, a small disturbance was successively added to each state variable and input variable here (while, other state variables and input variables remain unchanged) to calculate the output variables of the engine in the corresponding state successively according to the nonlinear engine model. The linearized model of the engine in this state is

$$
\left\{\begin{array}{l}
\dot{x}=A x+B u, \\
y=C x+D u,
\end{array}\right.
$$

where the state variable is $x=\left[n_{L} n_{H}\right]^{T}-\left[n_{L} n_{H}\right]_{q}^{T}$, the control variable is $u=\left[w_{f} A_{8}\right]^{T}-\left[w_{f} A_{8}\right]_{q}^{T}$, the output variable is $y=\left[n_{L} n_{H} P_{25} P_{3} T_{3} T_{45} T_{5}\right]^{T} \stackrel{q}{-}\left[n_{L} n_{H} P_{25} P_{3}\right.$ $\left.\left.T_{3} T_{45} T_{5}\right]\right]_{q}^{T}$, and the subscript $q$ denotes the steady state. The $n \cdot n$ matrix $A$ is the system matrix, $n \cdot p$ matrix $B$ is the input matrix, $q \cdot n$ matrix $C$ is the output matrix, and $q \cdot p$ matrix $D$ is the transfer matrix, where $n, p$, and $q$ are the dimensions of $x, u$, and $y$, respectively.

2.2. Sensor Fault Model. Common sensor faults include drift, saturation, open circuit, and deviation. Single fault and multifault modes are the states in which one or more sensors fail, respectively. When the sensor is in the state of failure, the control system cannot receive real data of the measured physical quantity, but this will not affect the state of the engine. The measured output value of the sensor in the fault state can be simulated by adding a fault component $f_{s k}$ to the actual value of the corresponding physical quantity $y_{k}$. The system shown in equation (4) is discretized. It was assumed in this case that both the system noise $w$ and measured noise $v$ are uncorrelated white noise. After adding the fault component of the sensor, the linear model at the steady-state point is

$$
\left\{\begin{array}{l}
x_{k}=A_{k, k-1} x_{k-1}+B_{k-1} u_{k-1}+w_{k-1}, \\
y_{k}=C_{k} x_{k}+D_{k} u_{k}+v_{k}+f_{s k}, \\
E\left(w_{k}\right)=E\left(v_{k}\right)=0 E\left[w_{k}, v_{j}^{T}\right]=0, \\
E\left[w_{k} w_{j}^{T}\right]=Q_{k} \delta_{k j} E\left[v_{k} v_{j}^{T}\right]=R_{k} \delta_{k j} .
\end{array}\right.
$$


2.3. Fault Feature Extraction. When the engine is working in the steady state, the control variable $u_{k}=0$ and the entire system are driven by noise. The output response of the same fault mode is proportional to the fault excitation without including system noise or measurement noise. Therefore, the direction of the output response to the same fault mode is the same; the size of the output response is proportional to the fault excitation. A sensor was selected here as the standard sensor, and its measured value was taken as the horizontal axis. The measured value of the nonstandard sensor was taken as the vertical axis. The output response of faults of different sizes in the same fault mode is shown in Figure 2.

The control system sensors are independent of each other, so the output responses in the single fault mode are also independent of each other. The multifault mode can be decomposed into a linear superposition of corresponding single fault modes. The characteristics of the multifault mode can be decomposed into the vector sum of the corresponding single-fault mode characteristics. The effects of fault excitation were eliminated here by using the feature extraction of the same fault mode. This provided a foundation for subsequent establishment of the FDIA system.

\section{FDIA System Based on the Immune Fusion Kalman Filter}

The application of Kalman filters and artificial immune systems in fault diagnosis and the shortcomings inherent to these techniques are described in this section. The immune fusion Kalman filter and the FDIA system based on it are then presented in detail.

3.1. Kalman Filter. The principle and calculation method under Kalman filter theory are discussed extensively in the literature [27-31]. For the linear system shown in equation (5), the prediction equation and state update equation of the Kalman filter are shown in equation (6) without considering the sensor fault:

$$
\left\{\begin{array}{l}
\hat{x}_{k / k-1}=A_{k, k-1} \widehat{x}_{k-1}+B_{k-1} u_{k-1}, \\
\widehat{x}_{k}=\widehat{x}_{k / k-1}+K_{k}\left(y_{k}-D_{k} u_{k}-C_{k} \widehat{x}_{k / k-1}\right), \\
K_{k}=P_{k / k-1} H_{k}^{T}\left(H_{k} P_{k / k-1} H_{k}^{T}+R_{k}\right)^{-1}, \\
P_{k / k-1}=A_{k, k-1} P_{k-1} A_{k, k-1}^{T}+Q_{k-1}, \\
P_{k}=\left(I-K_{k} H_{k}\right) P_{k / k-1} .
\end{array}\right.
$$

Reference [32] introduces the application of a bank of Kalman filters for aircraft engine fault diagnosis. The construction of the FDIA system based on the Kalman filter bank is shown in Figure 3. The state-space model, controller output, and sensor measurement are formed together as the input of the Kalman filter bank. Fault isolation is accomplished by comparing different WSSR (weighted sum of squared residual) values. The fault mode message is used as the decision variable to select the outputs of the Kalman filter bank. The selected reconstruction value is entered into the controller as input parameters and used to calculate the output of the controller.

It is necessary to design the corresponding Kalman filter for each potential fault to conduct effective control system fault diagnosis. The fault pattern was matched by the residual between the system output and the Kalman filter bank estimation. The number of Kalman filters that need to be designed at a steady-state point increases rapidly as the control system's output parameters increase. The diagnosis system cannot function properly under these conditions when fault occurs in the nondesign state based on the designed Kalman filter bank.

3.2. Artificial Immune System. The basic principles and algorithms of the AIS are discussed in detail in references [17-23]. The AIS was operated in a four-step process to estimate the state of the control system. First, the dimensions of the system were determined, and the detectors in the antibody library were initialized to fill as much of the state space as possible. The affinity function was set, and then, the affinity between the samples in the training set and the antibodies in the antibody library were calculated in turn to gradually mature the antibody library through multiple selection strategies (positive, negative, and clonal). The matured antibody library partitions the state space of the system. The system corresponds to different areas in the space under different working states; these areas are, respectively, occupied by antibodies representing their respective states. The antibody library evaluation operator was then introduced to determine whether the antibody library was mature. The antibody library was initialized and trained again if the calculation requirements were not met. Finally, the mature antibody library was used to detect the samples to be tested and diagnose the corresponding faults. A flow chart of this process is given in Figure 4.

The advantage of using the AIS in fault diagnosis is that the antibody library training reveals all the failure modes. The fault matching mode via the Kalman filter bank is not necessary. Algorithm redundancy and antibody cross-mutation strategies allow the FDI system to detect failure modes that are different from the training sample, which increases the stability of the system when encountering unknown failures. This approach also gives the system a certain evolution and self-learning ability.

The AIS is completely unknown to the failure mode and working mechanism of the system to be tested which can be regarded as a black box. The antibodies in the antibody library must fill as much of the system space as possible so that all states of the FDI system can be matched. The number of antibodies required by the AIS antibody library is generally $10^{n} ; n$ is the dimension of the system to be tested. When the dimension of the system to be tested is small (usually $n \leq 3$ ), the AIS can effectively diagnose the sample being tested. However, for the type of the control system investigated in this study, there are often more than seven states to be tested. The scale of the AIS antibody library is oversized in these cases, the calculation process is cumbersome and complicated, and the AIS is no longer applicable. 


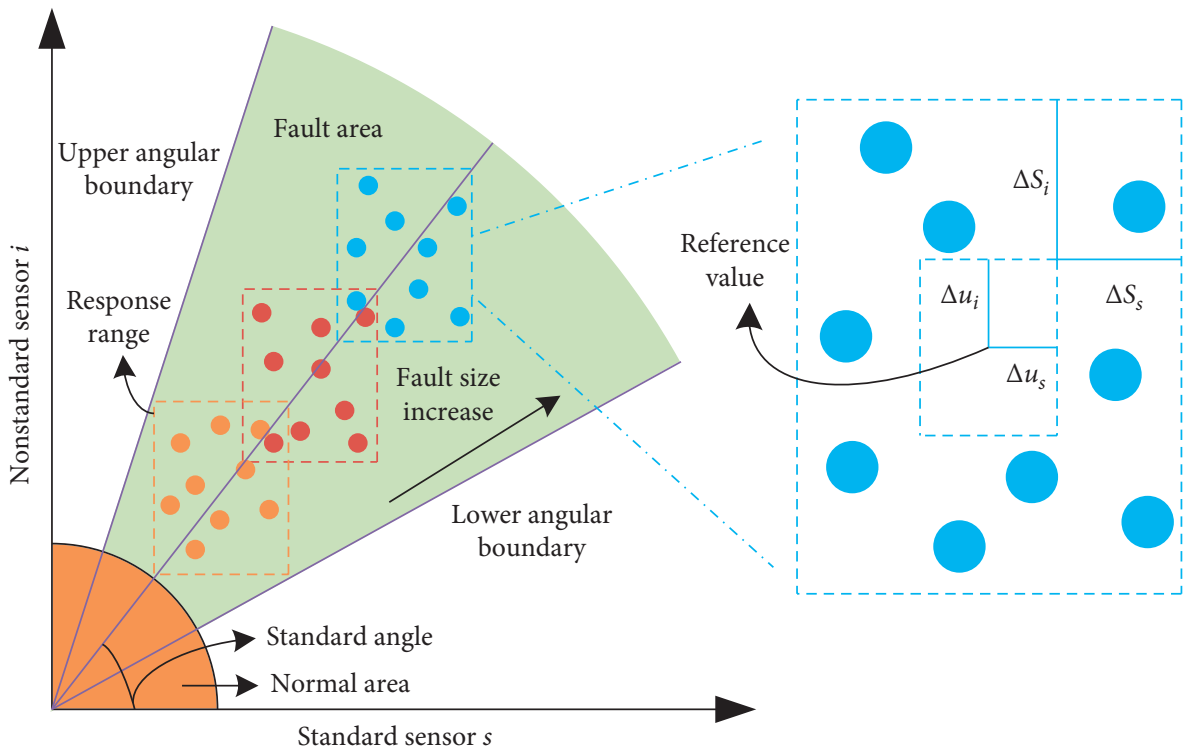

Figure 2: Fault feature extraction.

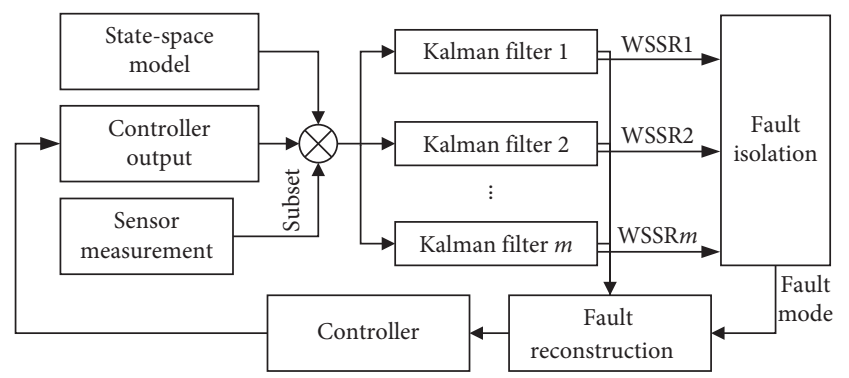

FIgURE 3: FDIA system based on the Kalman filter bank.

\subsection{FDIA System Based on the Immune Fusion Kalman Filter.} The immune fusion Kalman filter combines the Kalman filter's estimation of the system state with the artificial immune system's extraction of data features, which is wellsuited to the FDIA system for sensors in aero-engine control systems. The data flow of the FDIA system based on the immune fusion Kalman filter is shown in Figure 5. By introducing the nominal value of the model when the sensor fails and adding a specific noise model to train it, the FDIA system performs well even when the system is working under non-Gaussian noise. The failure mode of the system can be obtained by determining the affinity between an antibody and antigen for the purpose of fault isolation. The determined failure mode can then be reconstructed according to the immune optimization algorithm to obtain complete failure information. The immune fusion Kalman filter does not need a Kalman filter bank because it has an antibody library, which gives the system the ability to selflearn. When encountering a failure mode that is not in the training set during the diagnosis process, the antigen is split by redundancy and the antibody library is updated, while the failure mode is determined. Thus, the system can respond quickly when the corresponding failure occurs again. The

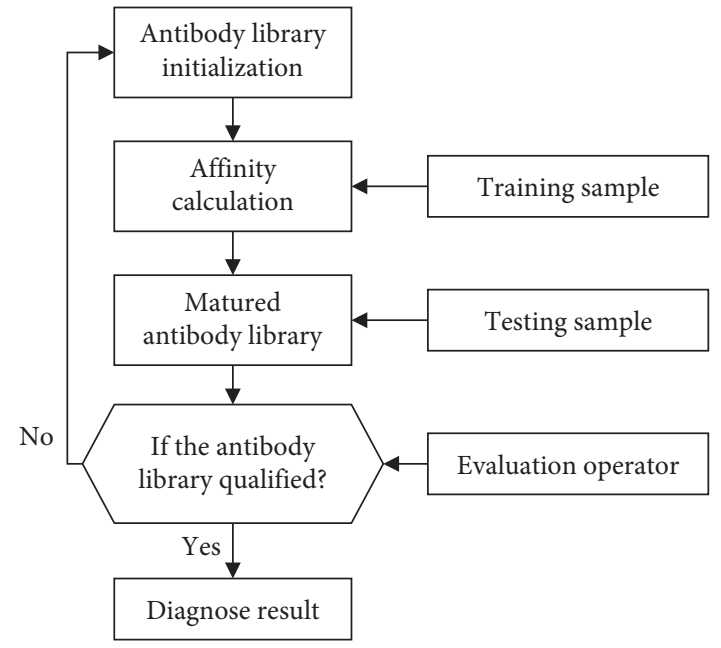

Figure 4: Fault diagnosis based on the artificial immune system.

estimation of the system state through the Kalman filter and the introduction of the nominal value of the fault during the training of the antibody library were found in this study to markedly reduce the scale of the antibody library and increase the calculation efficiency.

3.3.1. Antibody Library Training. For the FDI system based on artificial immune theory, each processed antigen is an input state of the diagnosis system. All possible input states of the system constitute the state space of the system. $U$ represents the set of all possible input states of the system, and $u$ represents the input status of the system. The $n$ dimension input state $u$ is normalized to set $U=[0,1]^{n}$ of all possible states of the system. The input state $u$ can be expressed as $u=\left[\begin{array}{llll}u_{1} & u_{2} & \ldots & u_{n}\end{array}\right]$, where $n$ is the number of 


\begin{tabular}{|c|c|c|c|c|}
\hline $\begin{array}{l}\text { Initialization } \\
\hat{x}_{0}=E\left[x_{0}\right] \\
P_{x 0}=E\left[\left(x_{0}-x_{0}\right)\left(\hat{x}_{0}-\hat{x}_{0}\right)^{T}\right] \\
\left\{\eta_{c}^{i}\right\}_{i=1}^{N} \geq 0.95 \\
\left\{\delta^{i}\right\}_{i=1}^{N} \quad \delta_{f} \\
a f_{s \max } \quad a f_{s \min }\end{array}$ & & $\begin{array}{l}\text { Kalman filter } \\
\hat{x}_{k / k-1}=A_{k, k-1} \hat{x}_{k-1}+B_{k-1} u_{k-1} \\
\hat{x}_{k}=\hat{x}_{k / k-1}+K_{k}\left(y_{k}-D_{k} u_{k}-C_{k} \hat{x}_{k / k-1}\right) \\
K_{k}=P_{k / k-1} H_{k}^{T}\left(H_{k} P_{k / k-1} H_{k}^{T}+R_{k}\right)^{-1} \\
P_{k / k-1}=A_{k, k-1} P_{k-1} A_{k, k-1}{ }^{T}+Q_{k-1} \\
P_{k}=\left(I-K_{k} H_{k}\right) P_{k / k-1}\end{array}$ & $\begin{array}{c}k=k+1 \\
x_{k}\end{array}$ & $\begin{array}{l}\text { Fault accommodation } \\
\text { Size }=a f_{s \min }: \text { step : } a f_{s \max }^{\text {Size }} \quad i \rightarrow\left\{a f_{s}^{\text {Size }}\right\} \\
\left\{a f_{s}^{\text {Size }}\right\} \rightarrow\left\{a f_{s j}^{\text {Size }}\right\}_{j=1}^{n p} \\
a f_{s j}^{\text {Size }}= \begin{cases}a f_{s \max } & a f_{s j}^{\text {Size }}>a f_{s \max } \\
a f_{s \min } & a f_{s j}^{\text {Size }}<a f_{s \min } \\
a f_{s j}^{\text {Size }} & \text { else }\end{cases} \end{array}$ \\
\hline$\nabla$ & & $x_{k}$ & & $d_{j}^{\text {Size }}=\sqrt{\sum_{m=1}^{n}\left(a f_{s j, m}^{\text {Size }}-a g_{m}\right)}$ \\
\hline $\begin{array}{l}\text { Antibody library training } \\
\left\{a b^{i}\right\}_{i=1}^{N}\end{array}$ & $\left\{a b_{k}^{i}\right\}$ & $\begin{array}{l}\text { Fault isolation } \\
a g=C_{k} \hat{x}_{k}+D_{k} u_{k}\end{array}$ & $i$ & $\operatorname{fit}\left(d_{j}^{\text {Size }}\right)= \begin{cases}f\left(d_{j}^{\text {Size }}\right) & d_{j}^{\text {Size }} \leq \delta_{f \mathrm{f}} \\
0 & d_{j}^{\text {Size }}>\delta_{f}\end{cases}$ \\
\hline$\left\{a b_{k}^{i}\right\}_{i=1}^{N} \quad n k$ & & $\begin{array}{l}\left.\operatorname{aff}\left(a g, a b_{k}^{i}\right)=1 / \sqrt{\sum_{m=1}^{n}\left(a g_{m}-a b_{k, m}^{i}\right.}\right)^{2} \\
\frac{1}{n} \sum_{k=1}^{n} \operatorname{aff}\left(a g, a b_{k}^{i}\right)>\delta^{i}\end{array}$ & & $\begin{array}{l}\mathrm{afit}^{\mathrm{Size}}=1 / n p \sum_{j=1}^{n p} \operatorname{fit}\left(d_{j}^{\text {Size }}\right) \\
\mathrm{ref}_{s}=\operatorname{Size} \mid \max \left\{\mathrm{afit}^{\mathrm{Size}}\right\} \\
\hat{x}_{k}=C_{k}^{-1}\left(y_{k}-\mathrm{ref}_{s}-D_{k} u_{k}\right)\end{array}$ \\
\hline
\end{tabular}

Figure 5: Data flow of the immune fusion Kalman filter.

input state components of the system and is related to the number of control system sensors.

After defining the self and nonself characteristics of the system, the set of inputs in all fault-free states is the self space represented by the set $S$. Similarly, the definition of the space that does not belong to the self space is the nonself space represented by $N_{s}$, where $N_{s}$ is the complement of the set $S$ on the full set $U$. For the aero-engine control system, the space $N_{f}$ formed by the fault state is a subset of $N_{s}$. The remaining part after $N_{s}$ delects $N_{f}$ is similar to the empty set, namely, $N_{s}-N_{f} \approx \varnothing$. The aero-engine control system sensor failure model was used in this study to obtain an antibody library $\left\{a b^{i}\right\}_{i=1}^{N}$ based on a single sensor failure, where $N$ is the number of control system sensors and $\left\{a b^{i}\right\}_{i=1}^{N}$ is the antibody library that is composed of nominal values. Sensor measurement noise in the actual engine process is unavoidable, so modeling error was also inevitable here. The antibodies in $\left\{a b^{i}\right\}_{i=1}^{N}$ were cloned to increase the robustness of the diagnostic system with Gaussian noise as an example.

The system established after adding state noise and measurement noise is shown in equation (5).

The output of the engine moves in a range near the nominal value of the system. The range of motion depends on the variance of the noise. For the normal distribution, most of the motion states are included in the interval $[-3 \sigma, 3 \sigma]$, so the $3 \sigma$ principle can also be used when considering the actual output motion range of the system. The cause of the actual system output deviation from the nominal value is composed of two parts. One part is caused by measurement noise, which is only related to the intensity of measurement noise; the other is caused by system state noise, which is not only related to the intensity of the system noise but is also related to the output response matrix $C$ of the system. The maximum offset of the measurement value of the $i$ th sensor due to measurement noise in this case is $\Delta s_{i}=3 \sigma_{i}^{s}$, where $\sigma_{i}^{s}$ is the standard deviation of the measurement noise of the $i$ th sensor. The maximum offset caused by system noise is $\Delta u_{i}=3 \sum_{j=1}^{n} C_{i j} \sigma_{j}^{u}$, where $C_{i j}$ is the element in the $i$ th row and $j$ th column of the system state-space equation output matrix, $n$ is the number of system state variables, and $\sigma_{j}^{u}$ is the standard deviation of the system noise corresponding to the $j$ th state variables. The nominal response of the system output and the motion range of the system response after considering the noise were determined here, as shown in Figure 2.

After determining the motion range of the system output response, the antibody was cloned in $\left\{a b^{i}\right\}_{i=1}^{N}$ so that the antibody library composed of the cloned antibody would cover the motion area of the system output response when a single sensor failed. The antibody coverage evaluation operator was introduced to evaluate the diversity of the antibody population. The antibody coverage can be calculated as follows:

$$
\eta_{c}^{i}=\frac{1}{S^{i}}\left(\sum_{k=1}^{n k} s_{k}^{i}+f_{i}\right)
$$

where $\eta_{c}^{i}$ is the antibody coverage evaluation operator corresponding to the $i$ th failure mode (usually above 0.95 ), $S^{i}$ is the area of the output response motion range when the $i$ th sensor fails, $s_{k}^{i}$ is the $k$ th antibody under the $i$ th failure mode, $n k$ is the scale of the antibody library after cloning, and $f_{i}$ is the sum of the area covered by multiple cloned antibodies in the $i$ th failure mode. Under the premise of a certain scale of the antibody library after cloning, greater antibody coverage remits better the adaptability of the antibody library to the output response in the fault state. The initialized antibody library $\left\{a b_{k}^{i}\right\}_{i=1}^{N}{ }_{k=1}^{n k}$ can be obtained after cloning selection of the antibody library $\left\{a b^{i}\right\}_{i=1}^{N}$. The initialized antibody library was found in this study to accurately match the output response of the system in the case of a single fault mode. 
3.3.2. Fault Diagnosis and Isolation. The input antigen of the immune system ag can be obtained from the Kalman filter's estimated value of the system state $\hat{x}_{k}$ combined with the system model. The affinity calculation function aff $\left(a g, a b_{k}^{i}\right)$ was set here to characterize the degree of similarity between $a g$ and the antibody in $\left\{a b_{k}^{i}\right\}_{i=1}^{N}{ }_{k=1}^{n k}$. A greater degree of similarity between the antigen and the antibody indicates greater corresponding affinity. The reciprocal of the Euclidean distance between the antigen and the antibody was selected as the affinity calculation function:

$$
\operatorname{aff}\left(a g, a b_{k}^{i}\right)=\frac{1}{\sqrt{\sum_{m=1}^{n}\left(a g_{m}-a b_{k, m}^{i}\right)^{2}}},
$$

where $m$ represents the $m$ th component of the corresponding antigen or antibody expression. The average affinity between $\mathrm{ag}$ and the antibodies in the same failure mode was calculated in $\left\{a b_{k}^{i}\right\}_{i=1}^{N}{ }_{k=1}$, and the corresponding affinity threshold $\delta^{i}$ was set according to the different failure modes. If the average affinity of $\mathrm{ag}$ to the $i$ th failure mode is greater than $\delta^{i}$, the control system is in the $i$ th single fault mode.

Different sensors are independent of each other in the control system sensor failure context. The output response when multiple sensors fail simultaneously can be decomposed into a linear combination of the output responses under the corresponding sensor single-fault state. The antibody corresponding to the multifault mode of the sensor can be decomposed into the vector sum of the antibody in the single fault mode of the corresponding sensor.

The failure excitations of different failure modes were different in the system under analysis here, so the range of movement of antibodies in any multifault mode was obtained by a combination of single fault antibodies in the antibody library. Through a series of antibody motion restriction ranges, the multifault mode was split to isolate the multifault mode. By restricting the antibody motion range, the diagnostic range based on the single failure mode antibody library was greatly expanded. After disassembling and isolating the input of the multifault mode encountered by each system, an antibody corresponding to the multifault mode was stored in the antibody library. When the input antigen of the same failure mode was encountered again, the immune fusion Kalman filter was able to respond quickly. Through continuous training and optimization of the antibody library, the antibody library was ensured to respond well to various common faults. The immune system was found to be strong enough to diagnose and isolate failure modes that had never been encountered during the training process.

3.3.3. Fault Accommodation Based on the Immune Optimization Algorithm. The role of fault accommodation is to deal with the known fault. For engine control-system sensor failure, fault accommodation is the estimation of the measured value of the faulty sensor, which is also called fault reconstruction. The fault reconstruction based on the immune optimization algorithm mainly includes three stages: the generation of the nominal antibody library based on the fault excitation, the optimization of the reconstruction antibody library, and the setting of the fitness function.

The first stage serves to establish a fault model of this fault mode based on the known fault mode and the linearized model of the engine at the steady-state point. The failure excitations were placed in order from small to large, and then, the nominal value of the system output under the action of different failure amplitude excitations in this failure mode was calculated to obtain the antibody library $\left\{a f_{s}^{\text {Size }}\right\}$ based on the nominal value of the failure amplitude. In the second stage, the reconstruction antibody library was optimized by a clonal selection process and a restriction selection process. Sensor measurement noise and the model error in the engine modeling process are inevitable in this case, so clone selection of the nominal value was used to obtain a reconstituted antibody library $\left\{a f_{s j}^{\text {Size }}\right\}_{j=1}^{n p}$ with an expanded population, where $n p$ is the scale of the antibody library after clonal selection. When the engine control system is in the $i$ th fault mode, the corresponding failure range is $\left[a f_{s \min } a f_{s \max }\right]$. However, in $\left\{a f_{s j}^{\text {Size }}\right\}_{j=1}^{n p}$, the antibody corresponding to the $i$ th fault mode may be $a f_{s j}^{\text {Size }} \notin\left[a f_{s \min } a f_{s \max }\right]$. The purpose of introducing the restriction selector was to correct the invalid antibodies in $\left\{a f_{s j}^{\text {Size }}\right\}_{j=1}^{n p}$ and maximize the computational efficiency of the antibody library.

The final stage is the setting of the fitness function, which mainly represents the antigen of the fault reconstruction system, that is, the estimated value of the output state of the system by the immune fusion Kalman filter and the matching degree of the antibody in $\left\{a f_{s j}^{\text {Size }}\right\}_{j=1}^{n p}$. Here, the Euclidean distance between the antigen of the fault reconstruction system and the antibody of $\left\{a f_{s j}^{\text {Size }}\right\}_{j=1}^{n p}$ was selected as the independent variable. The fitness function can be set as follows:

$$
\begin{aligned}
d_{j}^{\text {Size }} & =\sqrt{\sum_{m=1}^{n}\left(a f_{s j, m}^{\text {Size }}-a g_{m}\right),} \\
\operatorname{fit}\left(d_{j}^{\text {Size }}\right) & =\left\{\begin{array}{ll}
f\left(d_{j}^{\text {Size }}\right) & d_{j}^{\text {Size }} \leq \delta_{f} \\
0 & d_{j}^{\text {Size }}>\delta_{f}
\end{array},\right. \\
\text { afit }^{\text {Size }} & =\frac{1}{n p} \sum_{j=1}^{n p} \operatorname{fit}\left(d_{j}^{\text {Size }}\right),
\end{aligned}
$$

where $d_{j}^{\text {Size }}$ is the Euclidean distance between the antigen $a g$ and the reconstructed antibody $a f_{s j}^{\text {Size }}$, fit $\left(d_{j}^{\text {Size }}\right)$ is the fitness function with $d_{j}^{\text {Size }}$ as the independent variable, $\delta_{f}$ is the fitness function calculation threshold, and $\mathrm{afit}^{\mathrm{Size}}$ is the average fitness of the antibody group with an antigen corresponding to the failure excitation of Size. The calculated average fitness values of antigens and different fault excitations were arranged from large to small so that the fault excitation with the highest average fitness corresponded to the 
size of the current antigen's fault excitation. Finally, subtract the fault excitation from the measured value obtained by the sensor to reconstruct the state value $\hat{x}_{k}$ and return it to the Kalman filter. The fault reconstruction calculation process based on immune optimization is shown in Figure 6.

\section{Simulation and Analysis}

The simulation verification of the proposed FDIA system based on the immune Kalman filter is carried out under the MATLAB platform, and the result is compared with the Kalman filter bank and Kalman particle filter [7]. The simulation test consists of the following four parts:

(1) Performance evaluation of fault diagnosis and isolation

(2) Accuracy evaluation of parameter reconstruction

(3) FDIA system simulation under multifault conditions

(4) FDIA system simulation under transient conditions

Select the cruise working state under the engine standard environment state $(H=0 \mathrm{~km}$ and $\mathrm{Ma}=0)$ as state 1 . Correspondingly, let the maximum thrust state at high altitude ( $H=10 \mathrm{~km}$ and $\mathrm{Ma}=0.6)$ be state 2 . The input and output of the selected biaxial-separated exhaust turbofan engine in state 1 and state 2 are shown in Tables 1 and 2. Gaussian and non-Gaussian distribution systems were established according to the covariance of sensor measurement noise (Table 2). The probability density function of the Rayleigh distribution was calculated as

$$
f(x)= \begin{cases}\frac{x}{\sigma^{2}} \exp \left(\frac{-x^{2}}{2 \sigma^{2}}\right), & x>0, \\ 0, & x \leq 0 .\end{cases}
$$

The mean and variance of the Rayleigh distribution are $\sigma \sqrt{\pi / 2}$ and $(4-\pi) \sigma^{2} / 2$, respectively. According to the Rayleigh distribution, the noise has a nonzero mean value. To prevent the sensor fault reconstruction value of the control system from being affected by the nonzero mean value process, the probability distribution of the Rayleigh distribution was shifted to the left by $\sigma \sqrt{\pi / 2}$ along the $x$ axis.

The sensor faults of the aero-engine control system can be divided into hard faults and soft faults according to the failure mode. Among them, a hard fault is a fault that produces a large offset in an instant; this was realized here by adding a step function to the nominal value. When the sensor has a soft fault, the measured value of the sensor gradually deviates from the nominal value. This was realized by superimposing a ramp function on the basis of the nominal value during the simulation. There were 10 antibodies corresponding to the failure mode of each sensor in the antibody library of the immune fusion Kalman filter. The maximum magnitude of sensor failure was, by default, $20 \%$.

4.1. Fault Diagnosis and Isolation Simulation. The evaluation index of FDI system performance is mainly composed of accuracy rate, false alarm rate, and error rate. An accurate

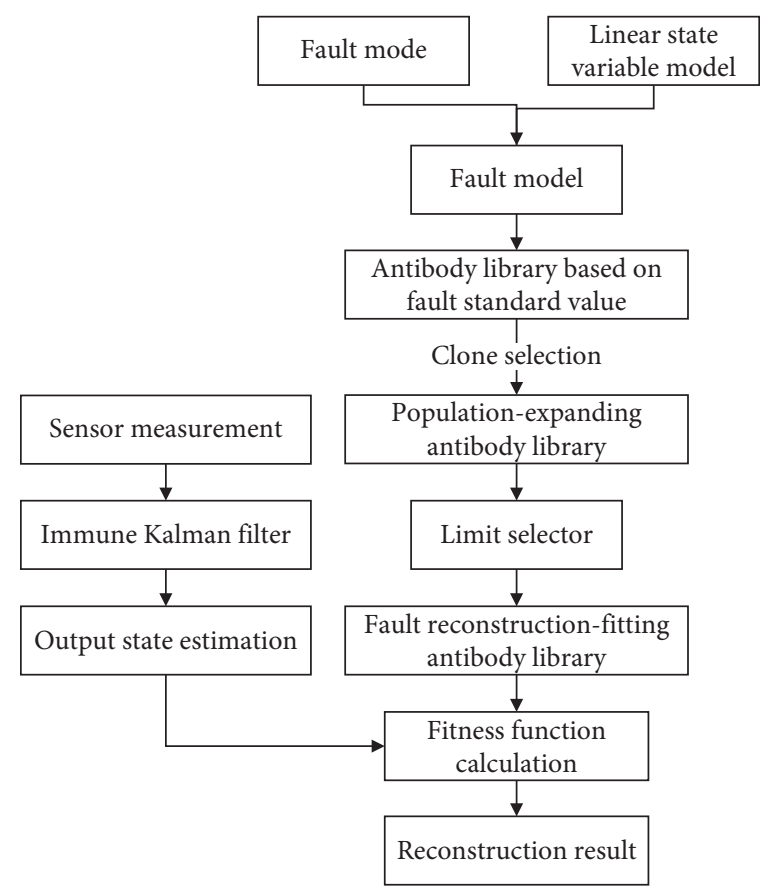

FIGURE 6: Fault reconstruction calculation process.

TABLE 1: Turbofan engine control variables and nominal values.

\begin{tabular}{lccc}
\hline Control variable & Symbol & State & Nominal value \\
\hline \multirow{2}{*}{ Engine oil } & \multirow{2}{*}{$W_{f}$} & 1 & $0.6305 \mathrm{~kg} / \mathrm{s}$ \\
\multirow{2}{*}{ Nozzle throat area } & \multirow{2}{*}{$A_{8}$} & 1 & $1.1135 \mathrm{~kg} / \mathrm{s}$ \\
\hline
\end{tabular}

TABle 2: Nominal value of turbofan engine measurement and sensor measurement error.

\begin{tabular}{lccc}
\hline Physical quantity & Measurement error & State & Nominal value \\
\hline$n_{L}$ & \multirow{2}{*}{0.0015} & 1 & $2324 \mathrm{RPM}$ \\
& \multirow{2}{*}{0.0015} & 1 & $4749 \mathrm{RPM}$ \\
\hline$n_{H}$ & \multirow{2}{*}{0.002} & 1 & $11370 \mathrm{RPM}$ \\
\hline$P_{25}$ & \multirow{2}{*}{0.002} & 2 & $178681 \mathrm{~Pa}$ \\
\hline$P_{3}$ & \multirow{2}{*}{0.0015} & 1 & $1106440 \mathrm{~Pa}$ \\
\hline$T_{3}$ & \multirow{2}{*}{0.0015} & 1 & $1392260 \mathrm{~Pa}$ \\
\hline$T_{45}$ & \multirow{2}{*}{0.0015} & 1 & $832.5 \mathrm{~K}$ \\
\hline$T_{5}$ & & 2 & $1423.6 \mathrm{~K}$ \\
\hline
\end{tabular}

diagnosis corresponds to the following two situations. The first is that when the system fails, the FDI system can accurately detect the fault and isolate the faulty component. On the contrary, when the system is working normally, the FDI system keeps quiet and does not issue any alarms. False 
alarm means that the FDI system issues a fault alarm when the detected system does not fail. When a wrong isolation occurs, it means that an error has occurred in the FDI system.

The measurement output of the engine in the fault state is mainly determined by environmental parameters $(H$ and $\mathrm{Ma})$, input parameters $\left(W_{f}\right.$ and $\left.A_{8}\right)$, and fault parameters (fault mode and fault size). The Monte Carlo method is used to quantitatively compare the performance of different FDI systems. The environmental parameters and input parameters jointly determine the state of the engine. A series of parameter combinations can be obtained by making small disturbances to the nominal values of environmental parameters and input parameters in a given state. Fault parameters are artificially set. The failure mode determines whether and which sensor fails. Corresponding to different types of faults, the physical meaning of the fault size is also different. In the hard fault mode, the fault size corresponds to the amplitude of the superimposed step signal. In soft fault simulation, the fault size is the slope of the ramp signal. The size of the fault mode is determined by the number of sensors in the engine control system. The fault size can be freely selected within the maximum value of the fault limit. By combining the fault modes and fault sizes, variety of fault parameters can be obtained.

The performance of different FDIA systems is evaluated from three aspects: correct rate, false alarm rate, and error rate by constructing different simulation environments by selecting different environmental parameters, input parameters, and fault parameters. Under the conditions of Gaussian noise and Rayleigh noise, 800 simulation experiments were carried out for each fault type (hard fault and soft fault). Results are shown in Tables 3 and 4.

The following conclusions can be drawn from Tables 3 and 4:

(1) The three methods can be divided into two categories, one is the traditional Kalman filter and the other is the ensemble method that combines the Kalman filter with other filtering methods. Under Gaussian noise, the accuracy of the three methods is roughly the same, and the correct rate of the FDIA system based on the Kalman filter bank is relatively high in some states. It can be concluded that, under Gaussian noise, both FDIA systems can diagnose and isolate sensor faults well.

(2) Under Rayleigh noise, the diagnosis accuracy rate based on the ensemble method is significantly higher than that of the Kalman filter bank. It can be proved that, after combining the Kalman filter with other methods, it can be used in the nonlinear condition. It is observed that, after training the antibody library with data based on a specific distribution, the FDIA system based on the immune fusion Kalman filter can still maintain a high diagnosis and isolation accuracy under non-Gaussian noise.

(3) The correct rate of soft faults is lower than that of hard faults. This is related to the selection of fault parameters. When the fault size is small, it is hard to diagnose the fault. Meanwhile, the soft fault is affected by fault size and fault time. Therefore, when fault size is small, soft faults are more difficult to diagnose than hard faults for the FDIA system. And, the correct rate for soft fault is also lower.

(4) The correct rate of working state 1 is generally higher than working state 2 . This is because, in the establishment of the engine nonlinear model, the model at state 2 is more nonlinear than state 1 . And, compared to state 1 , state 2 is more sensitive to disturbances. After linearizing the nonlinear model with the same method, the accuracy of the linearized model in working state 1 is higher than that in working state 2 , and the correct rate is also higher.

4.2. Fault Parameter Reconstruction Simulation. The quantitative evaluation index for fault parameter reconstruction is the average relative error (ARE) and the error standard deviation (ESD). ARE is the average value of the relative amount of difference between the reconstructed value and the real value during the simulation. ESD can be obtained by calculating the standard deviation of the difference between the reconstructed value and the true value. The calculation method of ARE and ESD is shown in the following formula where $y_{s}$ and $y_{r}$, respectively, correspond to the sensor measurement value and the actual value of the output parameter:

$$
\begin{aligned}
& \mathrm{ARE}=\frac{1}{n s} \sum_{n=1}^{n s}\left(\frac{\left|y_{s}-\mathrm{ref}_{s}-y_{r}\right|}{y_{r}}\right)_{n}, \\
& \mathrm{ESD}=\frac{1}{n s} \sqrt{\sum_{n=1}^{n s}\left[\left(\frac{\left|y_{s}-\mathrm{ref}_{s}-y_{r}\right|}{y_{r}}\right)_{n}-\mathrm{ARE}\right]^{2} .}
\end{aligned}
$$

Under different noise conditions, simulation experiments of hard fault and soft failure were performed on working states 1 and 2 . In the hard fault simulation, a $10 \%$ amplitude fault of $T_{3}$ sensor was added at $3 \mathrm{~s}$ to last for $4 \mathrm{~s}$. Figures 7-10 show the relationship between the measured value and the reconstruction value which is calculated under different methods. In the soft fault simulation, a fault with the slope $0.03 / \mathrm{s}$ of $n_{L}$ was added at $3 \mathrm{~s}$ to last for $4 \mathrm{~s}$. The relationship between the reconstruction value and standard value is shown in Figures 11-14. The results of ARE and ESD are shown in Tables 5 and 6, respectively.

It can be seen from the figure that, in the Gaussian process, the reconstruction accuracy based on the reconstruction results of the three methods is very high.

From Figures 7, 8, 11, and 12, it is observed that, under the Gaussian process, the reconstruction value of the fault sensor calculated by the immune fusion Kalman filter, Kalman filter bank, and Kalman particle filter matches the real value. Taking the noise type as the standard, compare Figures 9, 10, 13, and 14 to know that, in the non-Gaussian process, the Kalman filter bank could not track the measured value of the fault sensor well. The change trends of the reconstruction value and actual value were in accordance, 
TABLE 3: Simulation results of FDIA systems under Gaussian noise.

\begin{tabular}{|c|c|c|c|c|c|c|}
\hline Fault type & Method & State & Correct & False alarm & Error & Correct rate \\
\hline \multirow{6}{*}{ Hard fault } & \multirow{2}{*}{ Immune fusion Kalman filter } & 1 & 786 & 12 & 2 & 98.3 \\
\hline & & 2 & 784 & 13 & 3 & 98.1 \\
\hline & \multirow{2}{*}{ Kalman filter bank } & 1 & 788 & 9 & 3 & 98.5 \\
\hline & & 2 & 783 & 12 & 5 & 97.9 \\
\hline & \multirow{2}{*}{ Kalman particle filter } & 1 & 787 & 9 & 4 & 98.3 \\
\hline & & 2 & 785 & 9 & 6 & 98.1 \\
\hline \multirow{6}{*}{ Soft fault } & \multirow{2}{*}{ Immune fusion Kalman filter } & 1 & 765 & 18 & 17 & 95.6 \\
\hline & & 2 & 760 & 17 & 23 & 95.0 \\
\hline & \multirow{2}{*}{ Kalman filter bank } & 1 & 762 & 19 & 19 & 95.3 \\
\hline & & 2 & 756 & 21 & 23 & 94.5 \\
\hline & \multirow{2}{*}{ Kalman particle filter } & 1 & 768 & 19 & 13 & 96.0 \\
\hline & & 2 & 762 & 20 & 18 & 95.3 \\
\hline
\end{tabular}

TABLE 4: Simulation results of FDIA systems under Rayleigh noise.

\begin{tabular}{|c|c|c|c|c|c|c|}
\hline Fault type & Method & State & Correct & False alarm & Error & Correct rate $(\%)$ \\
\hline \multirow{6}{*}{ Hard fault } & \multirow{2}{*}{ Immune fusion Kalman filter } & 1 & 784 & 15 & 1 & 98.0 \\
\hline & & 2 & 782 & 14 & 4 & 97.8 \\
\hline & \multirow{2}{*}{ Kalman filter bank } & 1 & 534 & 40 & 226 & 66.8 \\
\hline & & 2 & 538 & 45 & 217 & 67.2 \\
\hline & \multirow{2}{*}{ Kalman particle filter } & 1 & 780 & 16 & 4 & 97.5 \\
\hline & & 2 & 784 & 13 & 3 & 98.0 \\
\hline \multirow{6}{*}{ Soft fault } & \multirow{2}{*}{ Immune fusion Kalman filter } & 1 & 761 & 23 & 16 & 95.1 \\
\hline & & 2 & 752 & 29 & 19 & 94.0 \\
\hline & \multirow{2}{*}{ Kalman filter bank } & 1 & 531 & 42 & 227 & 66.4 \\
\hline & & 2 & 514 & 48 & 238 & 64.3 \\
\hline & \multirow{2}{*}{ Kalman particle filter } & 1 & 758 & 24 & 18 & 97.6 \\
\hline & & 2 & 763 & 22 & 15 & 95.4 \\
\hline
\end{tabular}

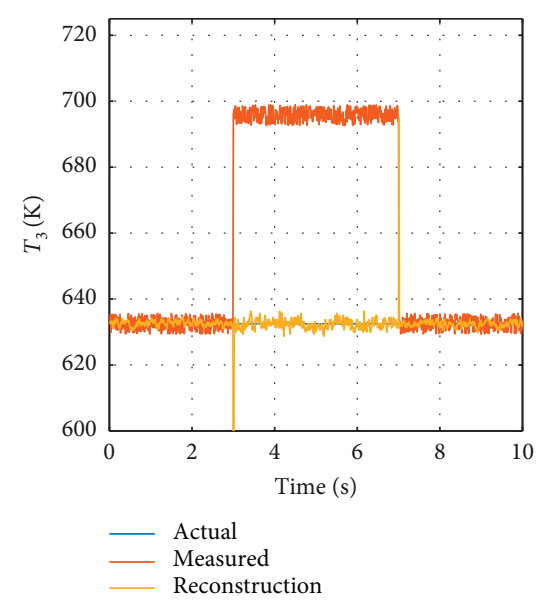

(a)

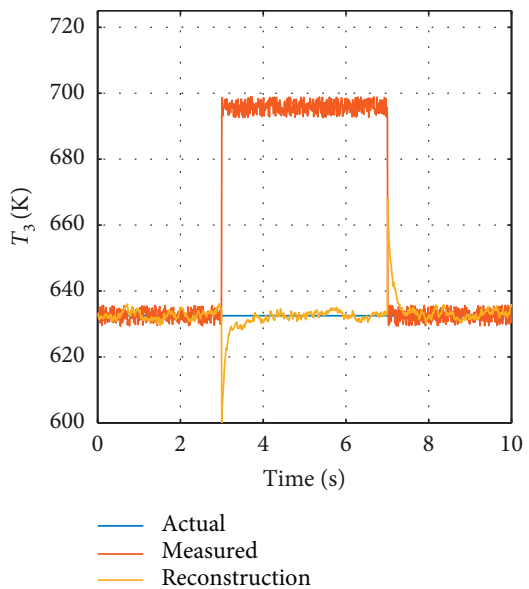

(b)

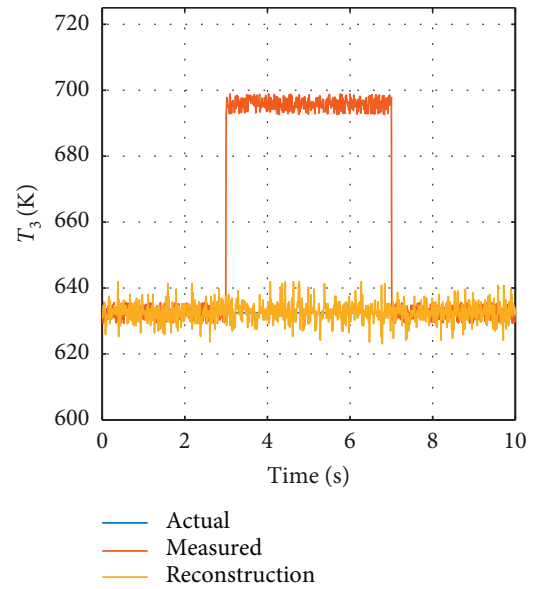

(c)

Figure 7: Reconstruction of $T_{3}$ sensor hard faults in state 1 under the Gaussian process. (a) FDIA system based on the immune fusion Kalman filter. (b) FDIA system based on the Kalman filter bank. (c) FDIA system based on the Kalman particle filter.

but the error between the corresponding time points was large. On the contrary, both the immune fusion Kalman filter and Kalman particle filter still performed high-precision reconstruction of the parameter that the fault sensor measured in the Rayleigh process. However, compared with the
Kalman particle filter, the reconstruction value calculated by the immune fusion Kalman filter is smoother and is less affected by noise. It is because unlike the particle filter, the artificial immune system is based on knowledge rather than the random process. The immune fusion Kalman filter can extend 


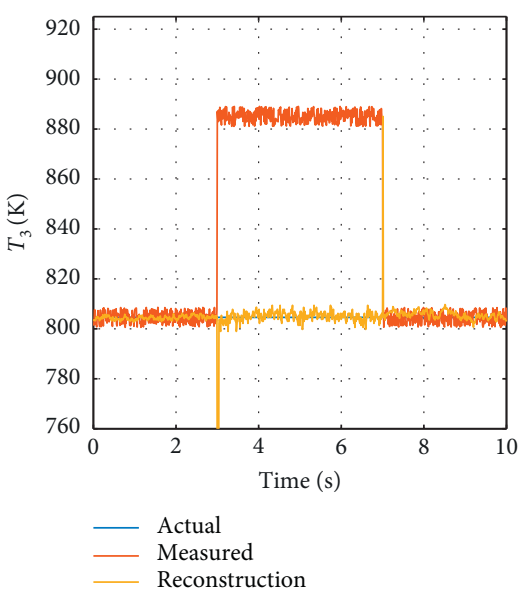

(a)

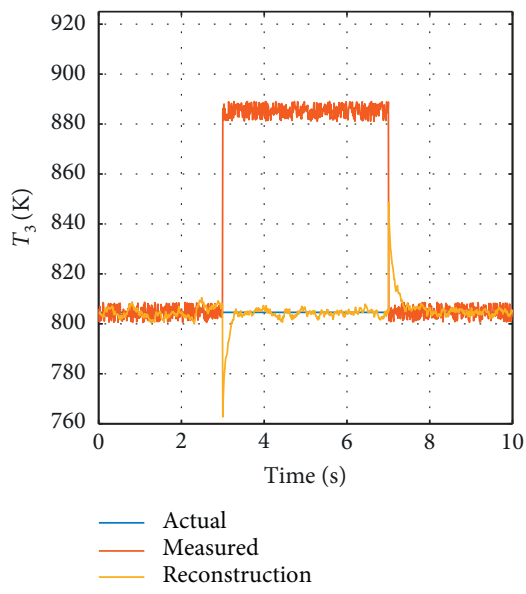

(b)

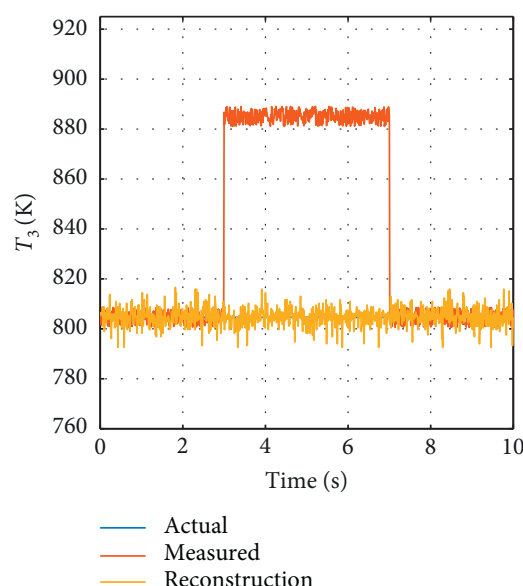

(c)

Figure 8: Reconstruction of $T_{3}$ sensor hard faults in state 2 under the Gaussian process. (a) FDIA system based on the immune fusion Kalman filter. (b) FDIA system based on the Kalman filter bank. (c) FDIA system based on the Kalman particle filter.

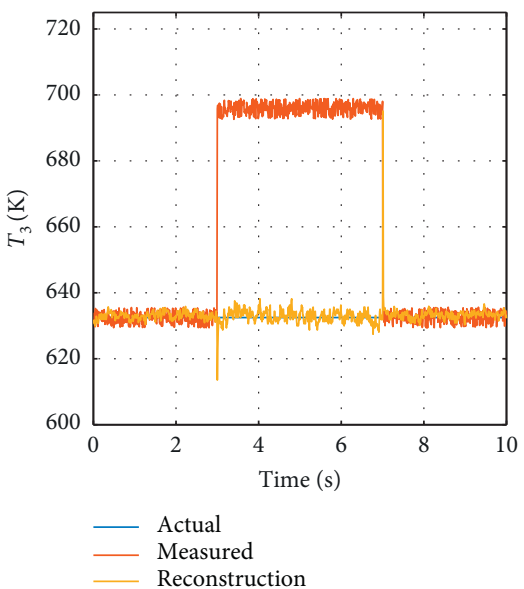

(a)

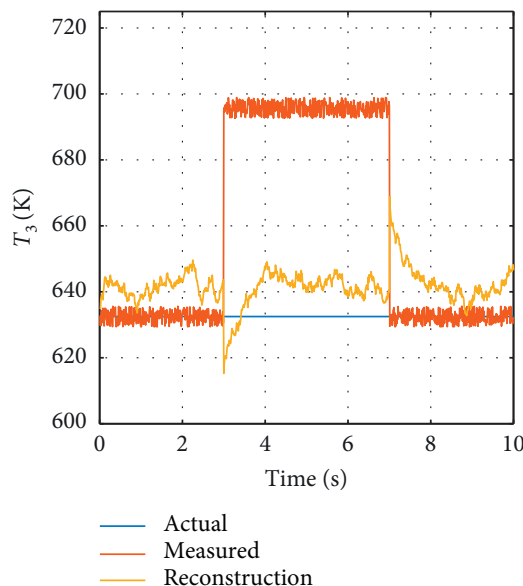

(b)

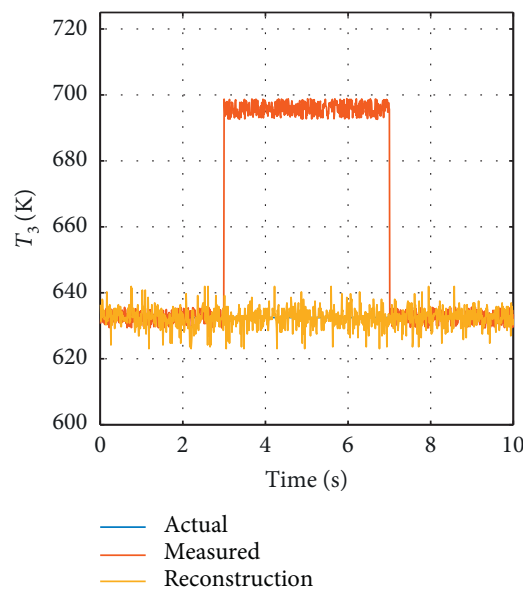

(c)

FIgURE 9: Reconstruction of $T_{3}$ sensor hard faults in state 1 under the Rayleigh process. (a) FDIA system based on the immune fusion Kalman filter. (b) FDIA system based on the Kalman filter bank. (c) FDIA system based on the Kalman particle filter.

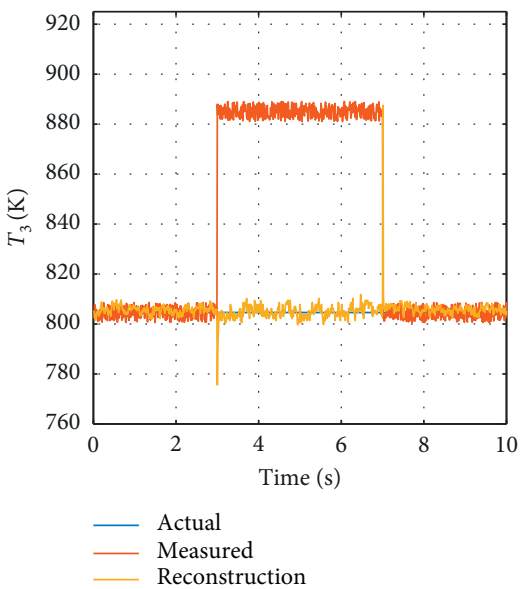

(a)

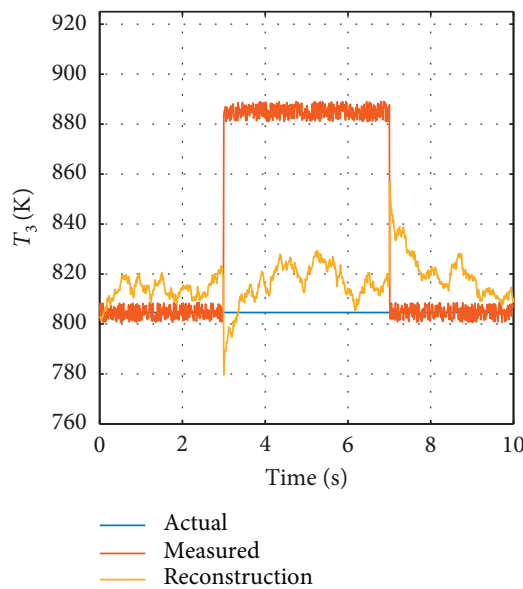

(b)

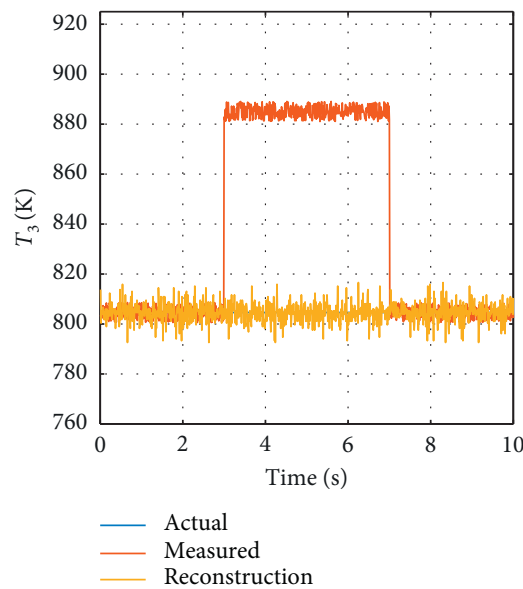

(c)

Figure 10: Reconstruction of $T_{3}$ sensor hard faults in state 2 under the Rayleigh process. (a) FDIA system based on the immune fusion Kalman filter. (b) FDIA system based on the Kalman filter bank. (c) FDIA system based on the Kalman particle filter. 


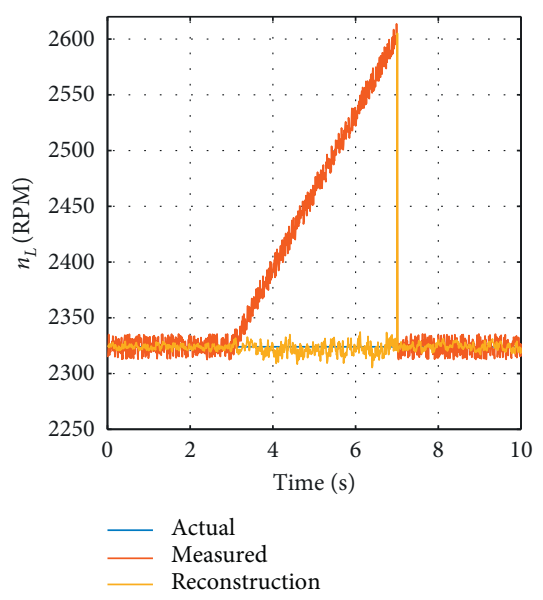

(a)

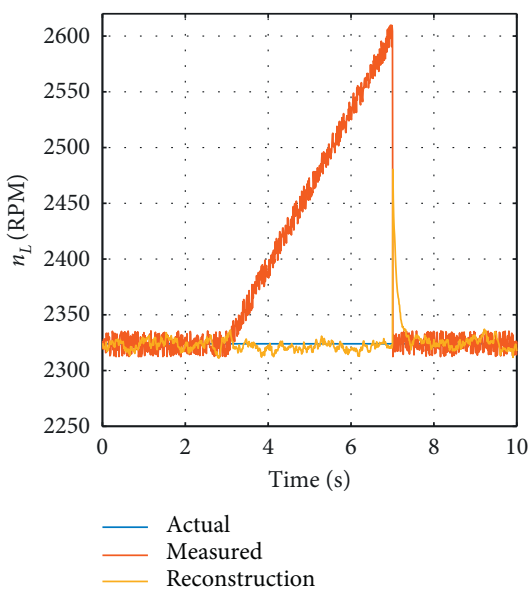

(b)

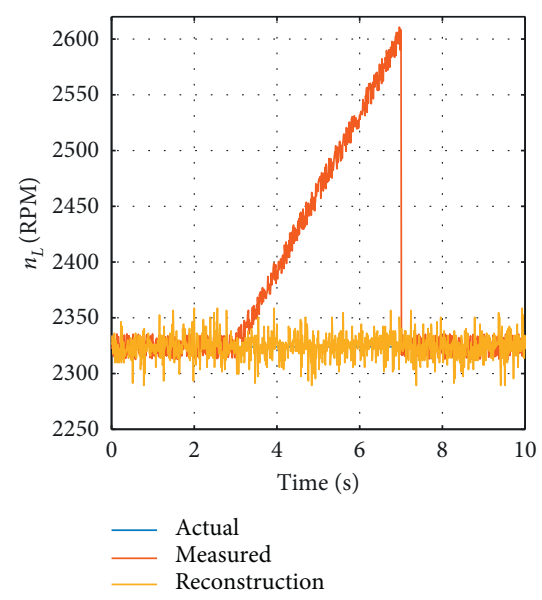

(c)

FIGURE 11: Reconstruction of $n_{L}$ sensor soft faults in state 1 under the Gaussian process. (a) FDIA system based on the immune fusion Kalman filter. (b) FDIA system based on the Kalman filter bank. (c) FDIA system based on the Kalman particle filter.

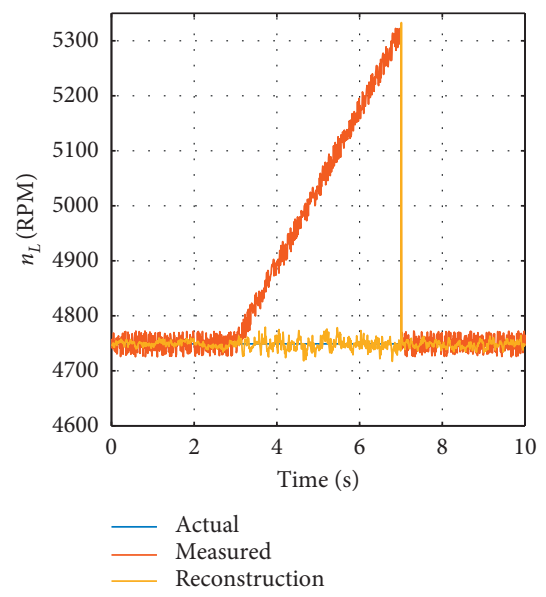

(a)

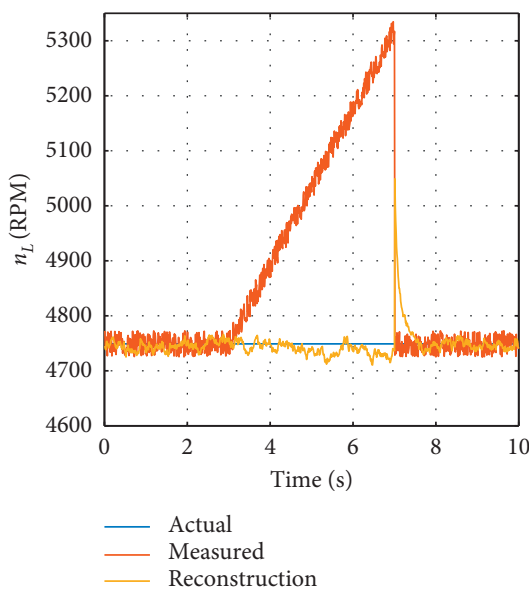

(b)

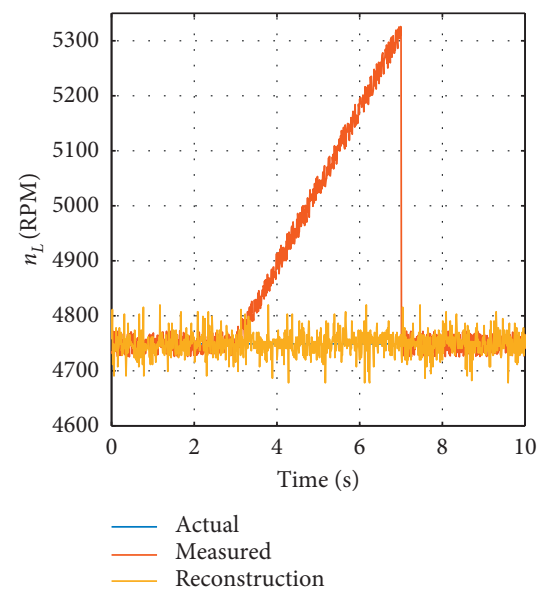

(c)

Figure 12: Reconstruction of $n_{L}$ sensor soft faults in state 2 under the Gaussian process. (a) FDIA system based on the immune fusion Kalman filter. (b) FDIA system based on the Kalman filter bank. (c) FDIA system based on the Kalman particle filter.

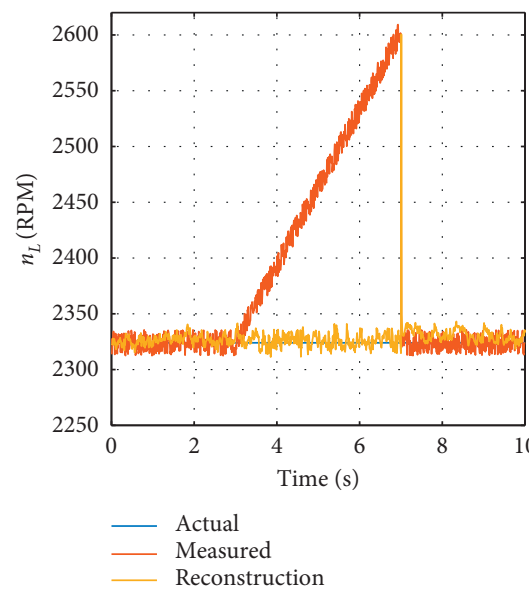

(a)

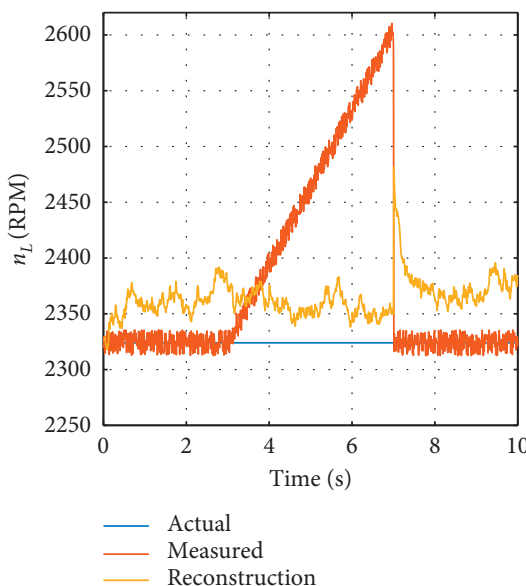

(b)

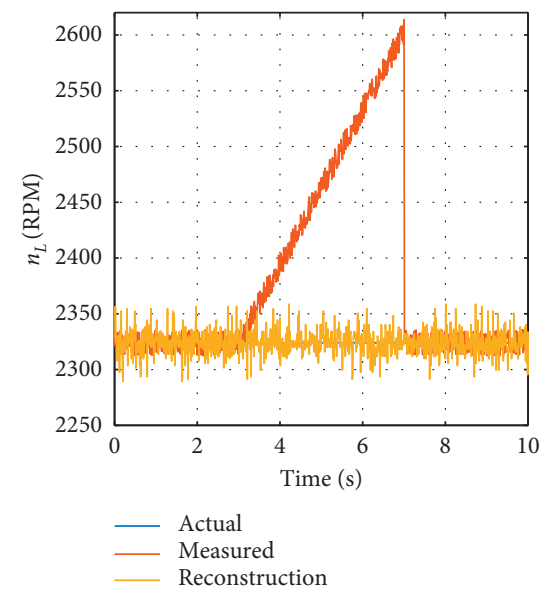

(c)

FIgURE 13: Reconstruction of $n_{L}$ sensor soft faults in state 1 under the Rayleigh process. (a) FDIA system based on the immune fusion Kalman filter. (b) FDIA system based on the Kalman filter bank. (c) FDIA system based on the Kalman particle filter. 


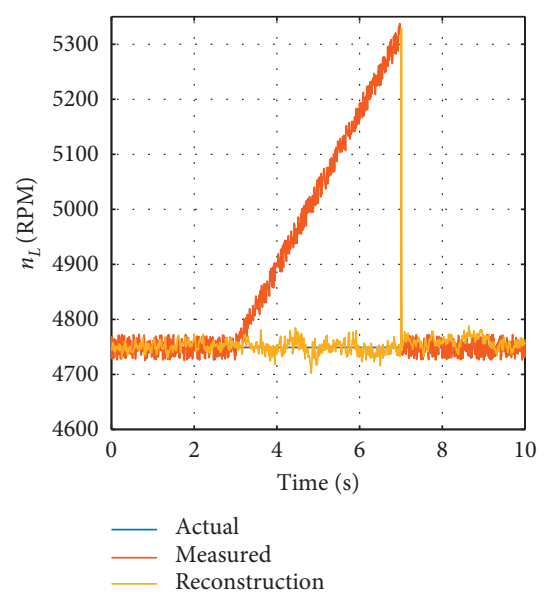

(a)

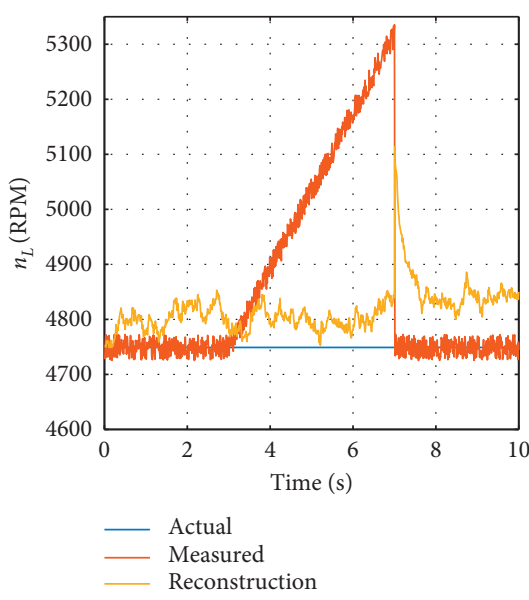

(b)

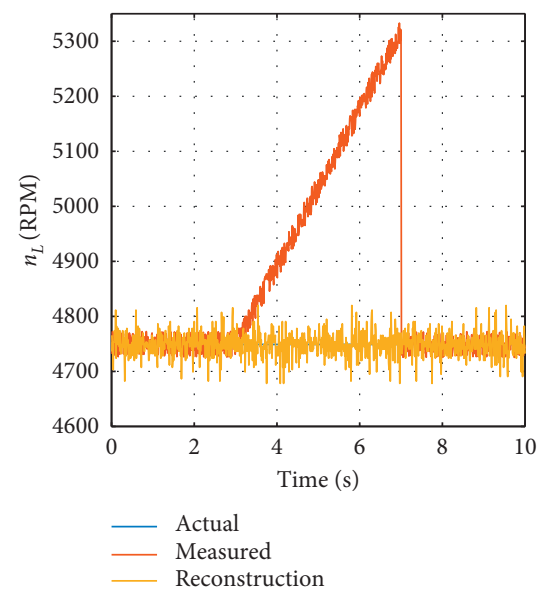

(c)

FIGURE 14: Reconstruction of $n_{L}$ sensor soft faults in state 2 under the Rayleigh process. (a) FDIA system based on the immune fusion Kalman filter. (b) FDIA system based on the Kalman filter bank. (c) FDIA system based on the Kalman particle filter.

TABLE 5: Evaluation table of fault reconstruction accuracy under hard fault conditions.

\begin{tabular}{|c|c|c|c|c|}
\hline Noise type & Method & State & $\operatorname{ARE}\left(10^{-2}\right)$ & $\operatorname{ESD}\left(10^{-5}\right)$ \\
\hline \multirow{6}{*}{ Gaussian } & \multirow{2}{*}{ Immune fusion Kalman filter } & 1 & 0.16 & 1.0129 \\
\hline & & 2 & 0.20 & 1.9958 \\
\hline & \multirow{2}{*}{ Kalman filter bank } & 1 & 0.26 & 2.6909 \\
\hline & & 2 & 0.26 & 2.6513 \\
\hline & \multirow{2}{*}{ Kalman particle filter } & 1 & 0.27 & 4.5847 \\
\hline & & 2 & 0.23 & 3.7956 \\
\hline \multirow{6}{*}{ Rayleigh } & \multirow{2}{*}{ Immune fusion Kalman filter } & 1 & 0.21 & 1.2607 \\
\hline & & 2 & 0.22 & 1.4082 \\
\hline & \multirow{2}{*}{ Kalman filter bank } & 1 & 1.52 & 4.5571 \\
\hline & & 2 & 1.58 & 7.6401 \\
\hline & \multirow{2}{*}{ Kalman particle filter } & 1 & 0.24 & 3.8233 \\
\hline & & 2 & 0.26 & 4.1701 \\
\hline
\end{tabular}

TABLE 6: Evaluation table of fault reconstruction accuracy under soft fault conditions.

\begin{tabular}{lcccc}
\hline $\begin{array}{l}\text { Noise } \\
\text { type }\end{array}$ & Method & State & $\begin{array}{c}\text { ARE } \\
\left(10^{-2}\right)\end{array}$ & $\begin{array}{c}\text { ESD } \\
\left(10^{-5}\right)\end{array}$ \\
\hline \multirow{6}{*}{ Gaussian } & Immune fusion Kalman & 1 & 0.14 & 1.5689 \\
& filter & 2 & 0.14 & 1.6090 \\
& & 1 & 0.22 & 2.0812 \\
& Kalman filter bank & 2 & 0.25 & 1.9776 \\
& & 1 & 0.26 & 4.3721 \\
& Immune fusion Kalman & 1 & 0.28 & 4.9971 \\
Rayleigh & filter & 2 & 0.21 & 1.6840 \\
& Kalman filter bank & 1 & 1.73 & 5.0573 \\
& & 2 & 1.44 & 7.3132 \\
& Kalman particle filter & 1 & 0.24 & 3.1788 \\
& & 2 & 0.25 & 3.8695 \\
\hline
\end{tabular}

the adaptability of the Kalman filter to non-Gaussian noise without changing the filter performance under Gaussian noise.

As shown in Tables 5 and 6, ARE, simulated in state 1 and 2, calculated for three methods in the Gaussian process were both the same order of magnitude. The ESD of the immune fusion Kalman filter and Kalman filter bank was slightly smaller than that of the Kalman particle filter. Therefore, in the Gaussian process, all algorithms have similar reconstruction quality. When using the immune fusion Kalman filter or Kalman particle filter to build the FDIA system, the calculated values of ARE and ESD in the two noise environments are equivalent which means that, after combining the Kalman filter with other algorithms that are applicable to non-Gaussian noise, the combined filter can adapt to different kinds of noise. But under Rayleigh noise, the reconstruction accuracy of the Kalman filter bank will be greatly reduced. This shows that, by using data with different noise distributions to train the immune fusion Kalman filter, the FDIA system can enhance its robustness to different noises, expanding the working range of the system.

4.3. Multifault Simulation. Next, under Gaussian process conditions, a soft fault with a slope $0.03 / s$ of $n_{L}$ sensor was added at $3 \mathrm{~s}$ for $4 \mathrm{~s}$ and a hard fault with a $10 \%$ amplitude of $n_{H}$ sensor was added at $2 \mathrm{~s}$ for $6 \mathrm{~s}$. Figure 15 shows the relationship between the actual value, the measured value, 


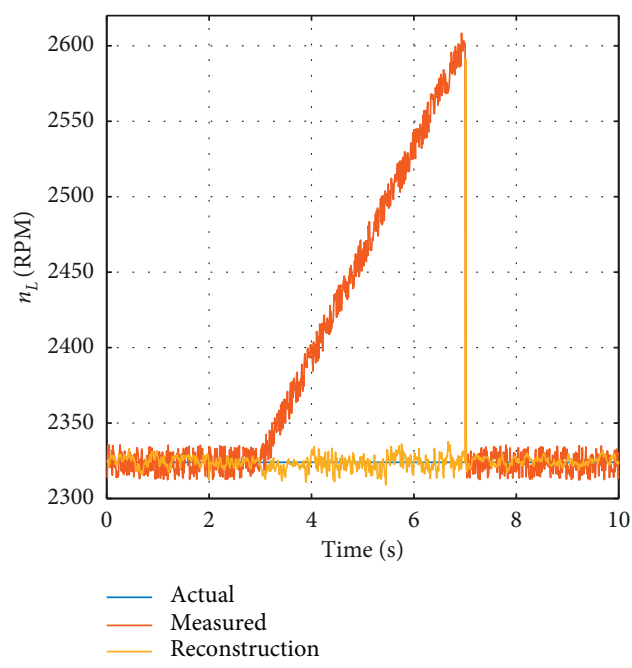

(a)

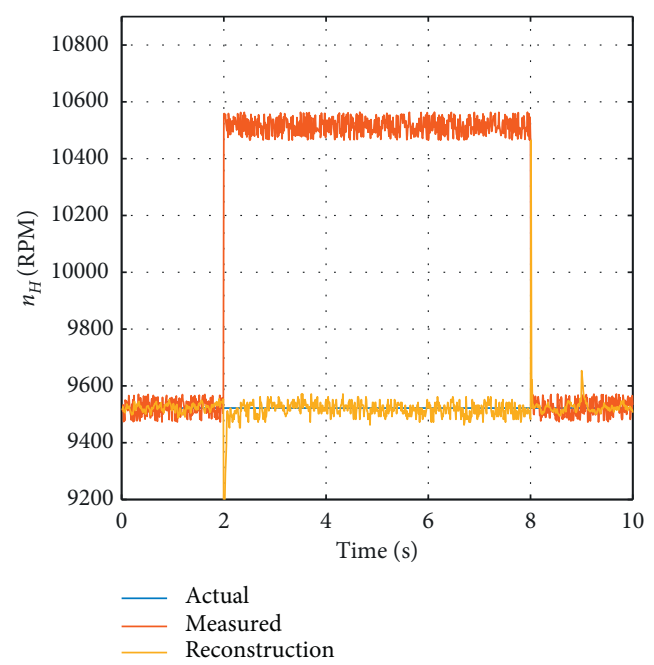

(b)

Figure 15: Reconstruction of multiple faults under the Gaussian process.

and the reconstruction value of $n_{L}$ and $n_{H}$, through the FDIA system based on the immune fusion Kalman filter.

Figure 15 shows that when multiple sensor failures occurred in the control system, the FDIA system based on the immune fusion Kalman filter split the multifault mode into independent fault modes and estimated the size of each mode. In this way, the reconstruction value of each parameter can be accurately obtained.

Compared with the Kalman filter bank that actually needs to be in advance, the FDIA system based on the immune fusion Kalman filter is simpler in structure and easier to adjust when dealing with multifault reconstruction problems.

4.4. Transient Simulation. Transient simulation compares the effects of FDIA systems designed by different methods on engine performance under sensor measurement failure conditions. In state 1 , the expected thrust of the engine during the simulation is shown in Table 7. Under Gaussian process conditions, a hard fault with a $10 \%$ amplitude of $n_{L}$ sensor was added at $20 \mathrm{~s}$ for $50 \mathrm{~s}$. Figures 16-18, respectively, show the relationship between thrust, $n_{L}$, and $n_{H}$ over time during the transient simulation. The transient simulation process is divided into the following four conditions:

(1) Expert condition: the relationship between the expected value of the corresponding physical quantity and time is determined by control expectation schedule

(2) Normal condition: the relationship between the measured value of the corresponding physical quantity and time is determined by the controller when control expectation schedule is certain

(3) Fault condition: relationship between the measured value of the corresponding physical quantity and time in the fault state
TABLe 7: Thrust control expectation schedule.

\begin{tabular}{lc}
\hline $\begin{array}{l}\text { Simulation time } \\
\text { period }(\mathrm{s})\end{array}$ & Thrust expectation \\
\hline $0-15$ & Maintain $12,000 \mathrm{~N}$ \\
$15-17$ & Linear change from $12000 \mathrm{~N}$ to $21000 \mathrm{~N}$ \\
$17-30$ & Maintain $21,000 \mathrm{~N}$ \\
$30-32$ & Linear change from $21000 \mathrm{~N}$ to $30000 \mathrm{~N}$ \\
$32-47$ & Maintain $30,000 \mathrm{~N}$ \\
$47-49$ & Linear change from $30000 \mathrm{~N}$ to $35000 \mathrm{~N}$ \\
$49-62$ & Maintain $35,000 \mathrm{~N}$ \\
$62-64$ & Linear change from $35000 \mathrm{~N}$ to $12000 \mathrm{~N}$ \\
$64-80$ & Maintain $12,000 \mathrm{~N}$ \\
\hline
\end{tabular}

(4) Reconstruct condition: When a sensor fails, the FDIA system reconstructs the physical quantity measured by the failed sensor and displays the reconstruction result over time.

As can be seen from Figure 16, compared with the Kalman filter bank and Kalman particle filter, the FDIA system based on the immune fusion Kalman filter has the fastest response speed and the highest reconstruction accuracy. When the sensor fails, with the reconstruction by the immune fusion Kalman filter, the parameter response of the engine in the fault state is consistent with the normal state, and it can greatly guarantee the performance of the engine and the safety of the aircraft. However, this performance improvement is only for fault conditions, and the entire control process is limited by the controller, and it cannot enhance the performance of the controller. Figures 17 and 18 show the reconstructed values of fault parameters and nonfault parameters, respectively. It can be seen from Figure 17, because $n_{L}$ sensor fails from $20 \mathrm{~s}$ to $50 \mathrm{~s}$, there is a strong jump at 20 seconds and 50 seconds in the fault condition, respectively. Since the FDIA system can reconstruct the fault value, it can reduce the deviation of the control effect caused by the sensor failure, and the FDIA system based on the immune fusion Kalman filter performs 


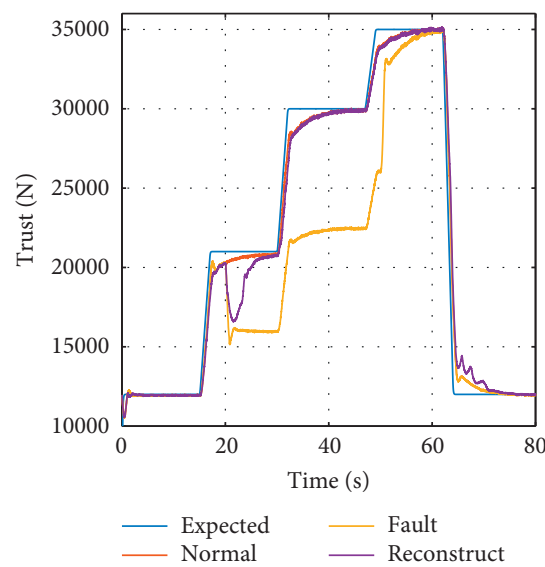

(a)

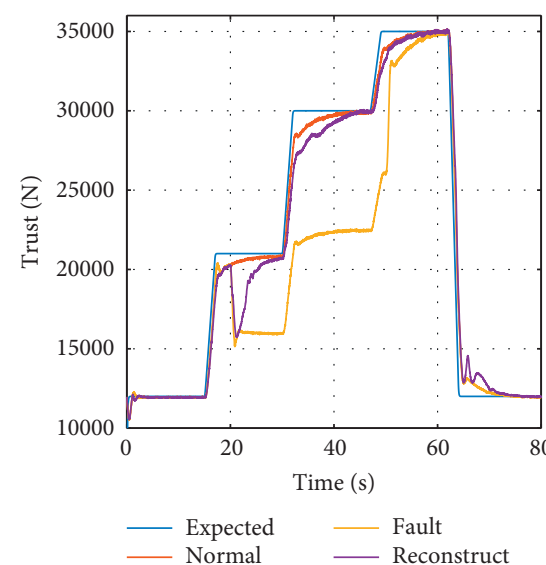

(b)

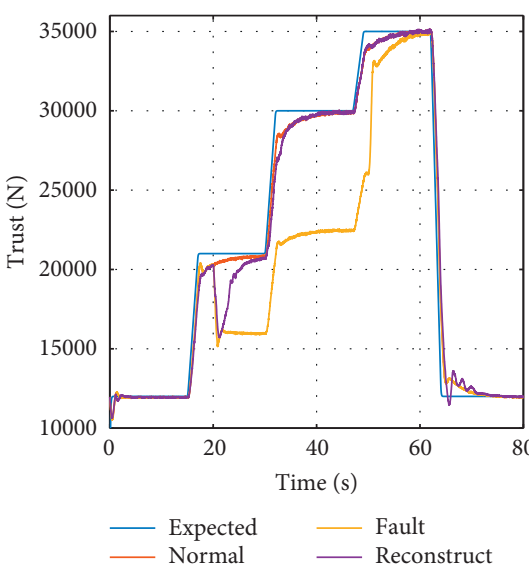

(c)

FIGURE 16: Relationship between trust and time when $n_{L}$ sensor malfunctions. (a) FDIA based on the immune fusion Kalman filter. (b) FDIA system based on the Kalman filter bank. (c) FDIA system based on the Kalman particle filter.

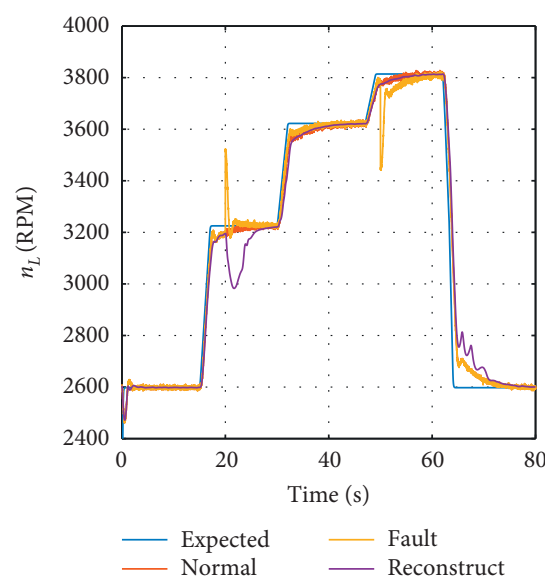

(a)

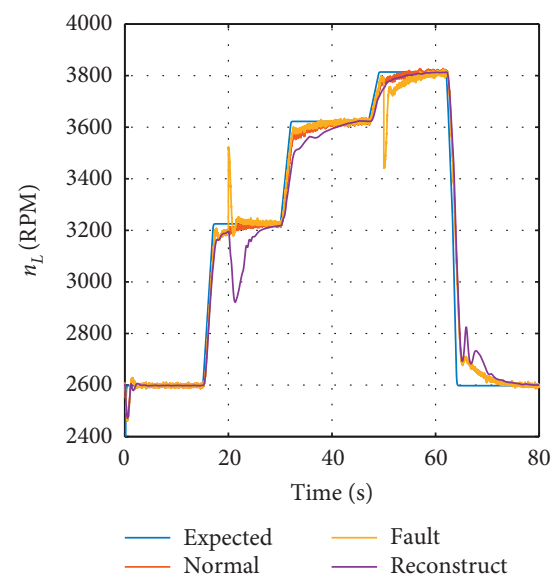

(b)

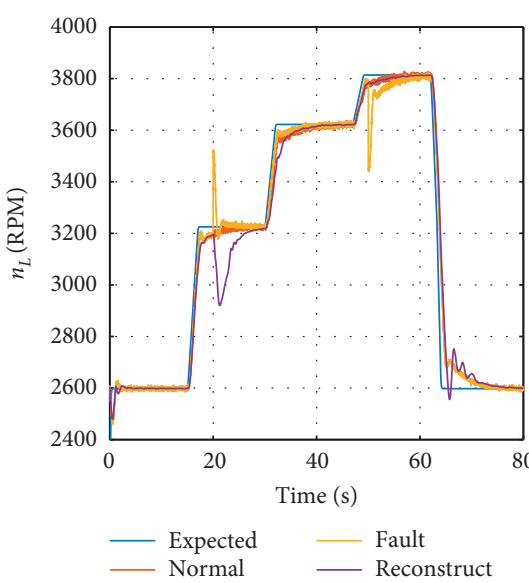

(c)

FIGURE 17: Relationship between $n_{L}$ and time when $n_{L}$ sensor malfunctions. (a) FDIA based on the immune fusion Kalman filter. (b) FDIA system based on the Kalman filter bank. (c) FDIA system based on the Kalman particle filter.

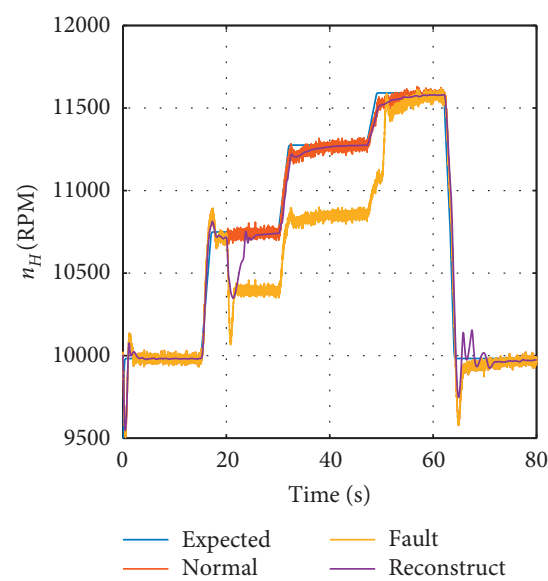

(a)

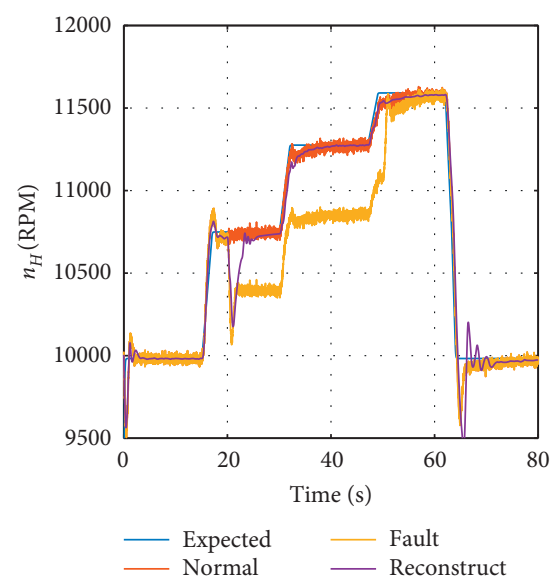

(b)

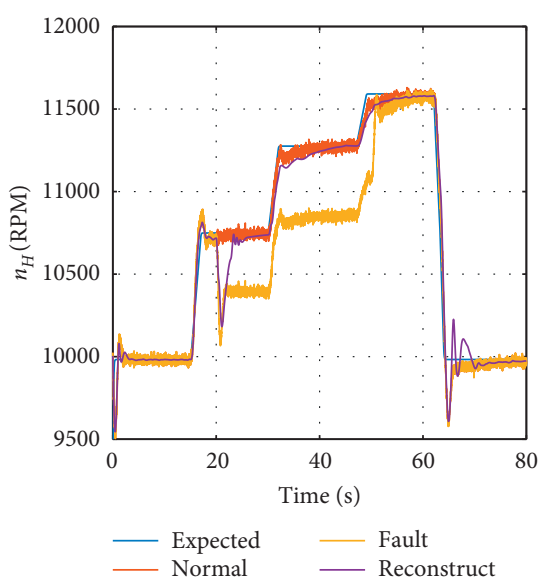

(c)

FIGURE 18: Relationship between $n_{H}$ and time when $n_{L}$ sensor malfunctions. (a) FDIA based on the immune fusion Kalman filter. (b) FDIA system based on the Kalman filter bank. (c) FDIA system based on the Kalman particle filter. 
best. From Figure 18, due to the failure of the sensor that measures the control parameters, the controller obtains the error information and gives the wrong output, which leads to the deviation of the no-fault parameters.

From Figures 16-18, it can be seen that the FDIA system enables the controller to obtain information that can reflect the actual state of the engine through reconstruction in the fault state, thereby eliminating the change in the engine working state caused by the sensor failure. From fault parameter reconstruction simulation, the immune fusion Kalman filter has the highest accuracy and smoothness; therefore, compared with the Kalman filter bank and Kalman particle filter, the FDIA system based on the immune fusion Kalman filter has the best control effect.

\section{Conclusion}

An innovative immune fusion Kalman filter was proposed in this study. Based on this method, a FDIA system for engine control system sensors is established. Simulations were conducted to show that the FDIA system based on the immune fusion Kalman filter effectively performs sensor fault reconstruction. Compared with the Kalman filter bank, the immune fusion Kalman filter has higher reconstruction accuracy and requires only a single filtering process to complete the diagnosis and isolation of faults, avoiding the complex multiple filtering. The antibody library concept was also introduced in the artificial immune system so that the immune fusion Kalman filter does not necessitate a KFbased fault matching filter bank. When facing multifaults, the FDIA system detects mutually independent single failure modes separately, and through the combination of the results, it can solve the isolation problem of multiple failure modes through the antibody library based on single failure mode training, which greatly increases the degrees of freedom of the system. In the non-Gaussian process, the FDIA system based on the immune fusion Kalman filter reconstructs the fault parameter with far greater accuracy than the Kalman filter bank. Compared with the other combined Kalman filter and Kalman particle filter, the immune fusion Kalman filter has strong scalability, and after having cooperated with the state extraction algorithm, it has greater potential for enhancing and guaranteeing aero engine performance. Theoretically, combined with the feature extraction algorithm, the immune fusion Kalman filter can deal with any fault that can be simulated by the nonlinear model, and it is also where the next research is going. The simulation results altogether prove that the immune fusion Kalman filter is capable of fault diagnosis, isolation, and accommodation and has broad potential application prospects.

\section{Data Availability}

The aero-engine control system sensor fault data used in this study are generated based on the model established in Section 2 of this paper. The engine model used to support the research results is currently under embargo for reasons of confidentiality. Requests for data, 6 months after publication of this article, will be considered by the corresponding author.

\section{Conflicts of Interest}

The authors declare that they have no conflicts of interest.

\section{Acknowledgments}

This work was supported by National Science and Technology Major Project (2017-V-0011-0062) and sponsored by the Seed Foundation of Innovation and Creation for Graduate Students in Northwestern Polytechnical University (CX2020013).

\section{References}

[1] R. B. Joly, S. O. T. Ogaji, R. Singh, and S. D. Probert, "Gasturbine diagnostics using artificial neural-networks for a high bypass ratio military turbofan engine," Applied Energy, vol. 78, no. 4, pp. 397-418, 2004.

[2] T. Kobayashi and D. L. Simon, "A hybrid neural networkgenetic algorithm technique for aircraft engine performance diagnostics," in Proceedings of the 37th AIAA/ASME/SAE/ ASEE Joint Propulsion Conference, Salt Lake City, UT, USA, July 2001.

[3] E. Naderi and K. Khorasani, "Data-driven fault detection, isolation and estimation of aircraft gas turbine engine actuator and sensors," Mechanical Systems and Signal Processing, vol. 100, pp. 415-438, 2018.

[4] L. Xiao, Y. Du, J. Hu, and B. Jiang, "Sliding mode fault tolerant control with adaptive diagnosis for aircraft engines," International Journal of Turbo \& Jet-Engines, vol. 35, no. 1, pp. 49-57, 2018.

[5] I. Yazar, F. Caliskan, and E. Kiyak, "Multiple fault-based FDI and reconfiguration for aircraft engine sensors," Aircraft Engineering and Aerospace Technology, vol. 89, no. 3, pp. 397-405, 2017.

[6] S. O. T. Ogaji, L. Marinai, S. Sampath, R. Singh, and S. D. Prober, "Gas-turbine fault diagnostics: a fuzzy-logic approach," Applied Energy, vol. 82, no. 1, pp. 81-89, 2005.

[7] F. Lu, J. Huang, and Y. Lv, "Gas path health monitoring for a turbofan engine based on a nonlinear filtering approach," Energies, vol. 6, no. 1, pp. 492-513, 2013.

[8] M. Kordestani, M. F. Samadi, M. Saif, and K. Khorasani, "A new fault prognosis of MFS system using integrated extended kalman filter and bayesian method," IEEE Transactions on Industrial Informatics, vol. 99, p. 1, 2018.

[9] M. Kordestani, M. Dehghani, B. Moshiri, and M. Saif, "A new fusion estimation method for multi-rate multi-sensor systems with missing measurements," IEEE Access, vol. 99, pp. 1-11, 2020.

[10] M. Kordestani, A. Chibakhsh, and M. Saif, "A control oriented cyber-secure strategy based on multiple sensor fusion," in Proceedings of the 2019 IEEE International Conference on Systems, Man and Cybernetics (SMC), Bari, Italy, October 2019.

[11] G. Chen and H. F. Zuo, "Expert systems of engine wear fault diagnosis based on knowledge rule," Journal of Aerospace Power, vol. 19, no. 1, pp. 23-29, 2004.

[12] Z. H. Wen and H. F. Zuo, "A diagnosis method for aero engine wear fault based on rough sets theory and integrated 
neural network," China Mechanical Engineering, vol. 18, no. 21, pp. 2580-2584, 2007.

[13] C. A. Laurentys, R. M. Palhares, and W. M. Caminhas, "Design of an artificial immune system based on danger model for fault detection," Expert Systems with Applications, vol. 37, no. 7, pp. 5145-5152, 2010.

[14] K. Ghosh and R. Srinivasan, "Immune system- inspired approach to process monitoring and fault diagnosis," Industrial and Engineering Chemistry Research, vol. 50, no. 3, pp. 1637-1651, 2010.

[15] S. Mohapatra, P.M. Khila, and R. R. Swain, "“Fault diagnosis in wireless sensor network using clonal selection principle and probabilistic neural network approach," International Journal of Communication Systems, vol. 32, p. 16, 2019.

[16] Q. Jiang and F. Chang, "A novel antibody population optimization based artificial immune system for rotating equipment anomaly detection," Journal of Mechanical Science and Technology, vol. 34, no. 9, pp. 3565-3574, 2020.

[17] J. Davis, M. G. Perhinschi, and H. Moncayo, "Evolutionary algorithm for artificial-immune-system-based failure-detector generation and optimization," Journal of Guidance, Control, and Dynamics, vol. 33, no. 2, pp. 305-320, 2010.

[18] H. Moncayo, M. G. Perhinschi, and J. Davis, "Artificial-immune-system-based aircraft failure evaluation over extended flight envelope," Journal of Guidance, Control, and Dynamics, vol. 34, no. 4, pp. 989-1001, 2011.

[19] D. A. Azzawi, M. G. Perhinschi, and H. Moncayo, "Artificial dendritic cell mechanism for aircraft immunity-based failure detection and identification," Journal of Aerospace Information Systems, vol. 11, no. 7, pp. 467-481, 2014.

[20] H. Moncayo, M. G. Perhinschi, and J. Davis, "Aircraft failure detection and identification using an immunological hierarchical multiself strategy," Journal of Guidance, Control, and Dynamics, vol. 33, no. 4, pp. 1105-1114, 2010.

[21] G. Mario, H. Moncayo, and J. Davis, "Integrated framework for artificial immunity-based aircraft failure detection, identification, and evaluation," Journal of Aircraft, vol. 47, pp. 1847-1859, 2010.

[22] M. G. Perhinschi, H. Moncayo, and D. Al Azzawi, "Integrated immunity-based framework for aircraft abnormal conditions management," Journal of Aircraft, vol. 51, no. 6, pp. 1726-1739, 2014

[23] G. Mario, J. Porter, H. Moncayo, J. Davis, and W. S. Wayne, "Artificial-immune-system-based detection scheme for aircraft engine failures," Journal of Guidance Control and Dynamics, vol. 34, pp. 1423-1440, 2011.

[24] J. G. Sun, V. Vasilyev, and B. Ilyasov, Advanced Multivariable Control Systems of Aeroengines, Bejjng University of Aeronautics \& Astronautics, Beijing, China, 2005.

[25] W. X. Zhou, Research on object-oriented modeling and simulation for aeroengine and control system, Ph.D. thesis, Nanjing University of Aeronautics and Astronautics, Nanjing, China, 2006.

[26] The Research and Technology Organization of NATO, Performance Prediction and Simulation of Gas Turbine Engine Operation for Aircraft, Marine, Vehicular; and Power Generation, NATO Research and Technology Organization, Paris, France, 2007.

[27] D. Simon and D. L. Simon, "Kalman filtering with inequality constraints for turbofan engine health estimation," IEEE Proceedings-Control Theory and Applications, vol. 153, no. 3, pp. 371-378, 2006.
[28] D. Simon, "A comparison of filtering approaches for aircraft engine health estimation," Aerospace Science and Technology, vol. 12, no. 4, pp. 276-284, 2008.

[29] Z. Qiu and L. Guo, "Improved Cubature Kalman filter for spacecraft attitude estimation," IEEE Transactions on Instrumentation and Measurement, vol. 70, 2020.

[30] A. J. Hoffman and N. Bester, "RSS and phase Kalman filter fusion for improved velocity estimation in the presence of real-world factors," IEEE Journal of Radio Frequency Identification, vol. 5, no. 1, 2020.

[31] Y. Huang, Y. Zhang, P. Shi, and J. Chambers, "Variational adaptive Kalman filter with Gaussian-inverse-Wishart mixture distribution," IEEE Transactions on Automatic Control, 2020.

[32] T. Kobayashi and D. L. Simon, "Application of a Bank of Kalman filters for aircraft engine fault diagnostics," in Proceedings of the International Joint Power Generation Conference, Atlanta, GA, USA, June 2003. 